$126 / 3179$

16. 212

LA-8040-MS

Informal Report

The GAMAMON Activation Library

MASTER

LOS ALAMO SCIENTIFIC LABORATORY

Post Office Box 1663 Los Alamo. New Mexico 87545 


\title{
The GAMMON Activation Library
}

\author{
M. E. Battat* \\ R. J. LaBauve \\ D. W. Muir
}

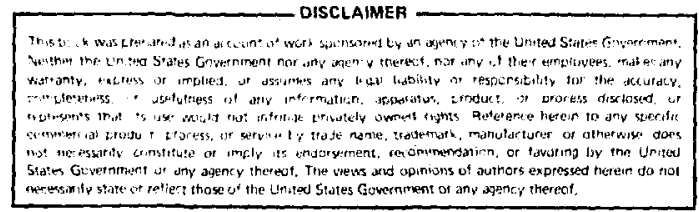

- LaSl Consultant.

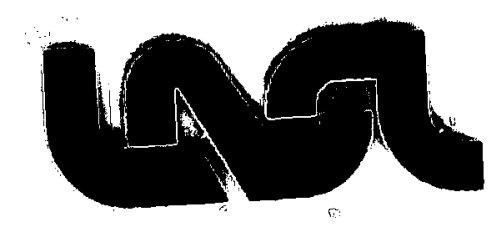


THE GAMMON ACTIVATION LIBRARY

\author{
by \\ M. E. Battat, R. J. LaBauve, and D. W. Muir
}

\begin{abstract}
This report describes the GAMMON activation library that is specifically designed for fusion reaction application but is also adequate for many other design studies. The library contains multigroup cross sections (in 100 energy groups) for 420 neutron-induced reactions, multigroup gamma-ray spectra (in 25 energy groups) for 107 unique daughter products, maximum permissible concentrations (MPC's) for 200 reaction products, and absorbable decay energy ("sensible heat") for 85 products.
\end{abstract}

\title{
I. INTRODUCTION
}

The GAMMON activation library contains neutron activation cross sections, radioactive decay information, maximum permissible concentrations (MPC), and total absorbable (gamma + non-gamma) energy release. The starting point for the GAMMON 1ibrary was the DLC-33/MONTAGE data file. ${ }^{1}$ The latter contains 100group neutron cross sections for 421 reactions and, where applicable, half-lives of the radioactive products. To this MONTAGE file we have appended the following information.

- MeV/dis of gamma radiation.

- $\quad$ ife $V / d i s$ of non-gamma radiation.

- For a gamma emitter, the photons per 100 disintegrations in a 25-group structure covering the energy range from 0 to $10 \mathrm{MeV}$.

- The MPC values as specified in the Code of Federal Regulations.

Before proceeding with a discussion of the data in the GAMMON file, some general comments would be in order. As with any data compilation, there will be as many "wish lists" for more information as there are users of the library. It is also certain that the choice of the number of groups and/or the energy group 
structure w111 not be universally compatible with the wide diversity of in-house user codes. More importantly, there will Inevitably be reactions of interest to some users that are not in the library. As a case in point, we are aware of certain reactions that are considered important for a particular TOKAMAK design ${ }^{2}$ and whilch are not in the GAMMON 11brary. Also, neutron cross sections for about 280 reactions in the file were calculated using the THRESH code; ${ }^{3}$ the use of this code was found to be conventent in the period prior to the inftial release of the MONTAGE 1fbrary in 1974. More sophisticated nuclear-model codes, for example, the GNASH code, ${ }^{4}$ are now avallable and should be employed in future updates and to replace any of the THRESH-generated cross sections in the GAMMON library that may be of practical interest. These admitted shortcomings exist in the library because of limited resources alloted to this task, but the library approaches theccurrent state of the art. Furthermore, we feel that, before embarking on another major revision of the 11brary, some consensus on what it should contain is necessary. However, this does not detract from the present usefulness of the library for fusion reactor calculations. With the addition of some user-supplied data for specific applications, the file should be adequate for most design studies. Any future update, revisions, and additions to the data should consider inputs from users so that the library can be useful to a Jarger number of the fusion-reactor community.

\section{NEUTRON CROSS SECTIONS}

The GAMMON file contains data for 420 reactions, " of which about 100 were processed using ENDF/B-IV data. Approximately 40 of the reaction cross sections were obtained from other sources (see Ref. 1) with the rest calculated using the THRESH code. Activation cross sections are given for 100 energy groups (the GAMII structure); the energy boundaries of the groups are listed in Table I. The weighting function is $1 / \mathrm{E}$ except near $14 \mathrm{MeV}$ where a thermally broadened $(T=20$ $\mathrm{MeV}$ ) fusion peak is employed. A listing of the reactions for which data are given, together with other relevant information, is shown in Table II. This

This is one less than the number of reactions in DLC-33/MONTAGE. We deleted the data for the $54 \mathrm{Fe}(\mathrm{n}, \gamma) 55 \mathrm{Fe}$ reaction because the cross-section behavior was incorrect, and the information could not be found in the reference cited for the data. A few typographical errors were also corrected, the main one being the group 100 cross section, 4.61 $\times 10^{-4}$ instead of $4.61 \times 10^{-40}$, for the ${ }^{2} \mathrm{H}(\mathrm{n}, \mathrm{\gamma})^{3} \mathrm{H}$ reaction. 
TABLE I

NEUTRON MULTIGROUP STRUCTURE

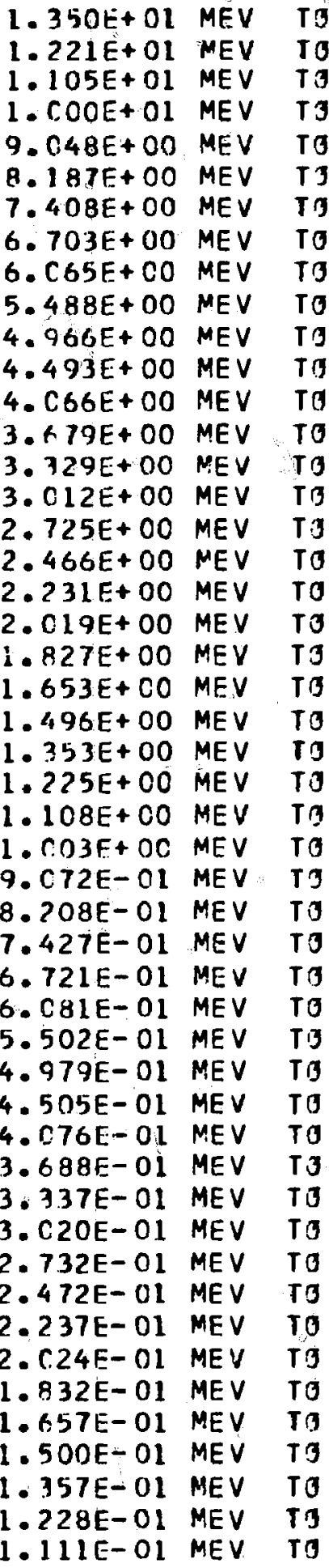

1.492E+01 MEV

1.35 OE+OL MEV

1.22 IE+ O1 MEV

1.105E+OL MEV

1. $000 \mathrm{E}+01 \mathrm{MEV}$

9. $048 E+00 \mathrm{MEV}$

8.187E + OO MEV

$7.408 E+00$ MEV

6. $703 E+00$ MEV

6.065E+ 00 MEV

5.48 8E+ 00 MEV

4.96 $6 E+00$ MEV

4.493E+ $00 \mathrm{MEV}$

4.06 $6 \mathrm{E}+00 \mathrm{MEV}$

3.679E+ OO MEV

3. $329 E+00^{\circ}$ MEV

3. $012 \mathrm{l}+00 \mathrm{MEV}$

$2.725 \mathrm{E}+00$ MEV

$2.466 \mathrm{E}+00 \mathrm{MEV}$

$2.231 E$ t OO MEV

2.019E 00 MEV

1.82 TE $\$ 00 \mathrm{MEV}$

1. $653 \mathrm{E}+00 \mathrm{MEV}$

1. $.496 E+00$ MEV

$1.353 E+$ DO MEV

1.225E+00 MEV

1. 10 BE + 00 MEV

1. $003 E+00 \mathrm{MEV}$

9.072E-O1 MEV

8.208E-OL MEV

7.42 7E-O1 MEV

6.72 IE-OI MEV

6.08 LE-01 MEV

5.502E- TL MEV

4.979E-OL MEV

4.505E-01 MEV

4.076E-O1 MEV

3.68 8E-01 MEV

3.337E-01 MEV

3. 02 CE-O1 MEV

2. $732 E-01$ MEV

2. $472 E-O 1$ MEV

2.23 7E-O1 MEV

2. $024 \mathrm{E}-01$ MEV

1. $832 \mathrm{E}-01 \mathrm{MEV}$

1. $.657 \mathrm{E}-01 \mathrm{MEV}$

1. $500 E-01$ MEV

1.357E-O1 MEV

1. $228 E-01$ MEV
1.420E+00 MEV

$1.285 E+00 \mathrm{MEV}$

$1.162 \mathrm{E}+00 \mathrm{MFV}$

1. $052 \mathrm{E}+00 \mathrm{MEV}$

$9.516 E-01$ MEV

8.611E-O1 MEV

$7.791 \mathrm{E}-01 \mathrm{MEV}$

7.050E-01 MEV

6.379E-O1 MEV

5.772E-O1 MEV

$5.223 E-01 \mathrm{MEV}$

4.726E-O1 MEV

4.276E-01 MEV

3.869E-01 MEV

3.501E-01 MEV

3.168E-01 MEV

2.866E-01 MEV

2.593E-01 MEV

$2.347 E-01$ MEV

$2.123 E-01$ MEV

$1.921 \mathrm{E}-01 \mathrm{MEV}$

1.738E-01 MEV

1.573E-01 MEV

1.423E-01 MEV

1.288E-01 MEV

1.165E-01 MEV

1.054E-01 MEV

9.541E-02 MEV

$8.633 E-02 \mathrm{MEV}$

7.81 IE-02 MEV

7.068E-02 MEV

$6.395 E-02$ MEV

5.787E-02 MEV

5.236E-02 MEV

4.738E-02 MEV

4.287E-02 MEV

3.879E-02 MEV

3.510E-02 MEV

3.176E-02 MEV

2.874E-02 MEV

2.600E-02 MEV

$2.353 E-02$ MEV

2.129E-02 MEV

$1.926 E-02$ MEV

1.743E-02 MEV

$1.577 E-02$ MEV

$1.427 E-02$ MEV

1.291E-02 MEV

1.168E-02 MEV 
TABLE I (contd.)

GROUP

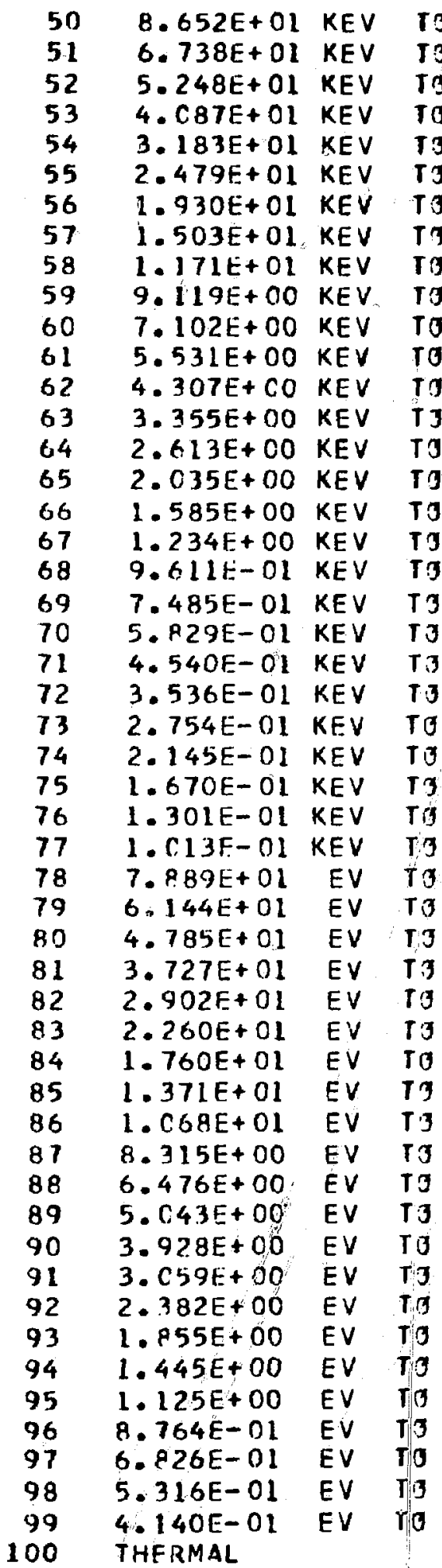

8.652E+ O1 KEV

6. $738 E+01$ KEV

5.24 8E+ 01 KEV

4.08 TE+01 KEV

3.183E+ OL KEV

2.479E+ 01 KEV

1.93 OE+DL KEV

1.503E+ O1 KEV

1. 17 LE+OI KEV

3.119E+00 KEV

7.102E+ 00 KEV

$5.531 E+00 \mathrm{KEV}$

4. $307 E+00 \mathrm{KEV}$

$3.355 E+00 \mathrm{KEV}$

$2.613 E+00 \mathrm{KEV}$

$2.035 E+00 \mathrm{KEV}$

I. $585 E+C O$ KEV

1. $234 \mathrm{E}+00 \mathrm{KEV}$

$9.611 F-01 \mathrm{KEV}$

7. $485 E-01 \mathrm{KEV}$

5. $829 E-01 \mathrm{KEV}$

4.54 CE-OI KEV

3.536E-OI KEV

2.754E-01 KEV

$2.145 E-01$ KEV

1.670E-O1 KEV

1.301E-01 KEV

1.013E-01 KEV

$7.889 E+01$ EV

$6.144 E+01$ EV

4. $.785 E+01$ EV

3.727E+01 EV

2. $902 E+01$ EV

2. $260 E+01$ EV

1.76OE+01 EV

1.371E+01 EV

$1.068 E+01$ EV

8.315E+00 EV

$6.476 E+00$

$5.043 E+00$

3. 9 ? $8 \mathrm{E}+00$

$3.059 E+00$

2. $382 E+00$

1. $855 E+00$

$1.445 E+00$

1. $125 E+00$

8. $764 E-01$

$6.826 E-01$

5. $316 E-01$
EV

EV

EV

EV

EV

EV

EV

EV

EV

EV

EV
ENERGY WIUTH

$2.457 E+01 \mathrm{KEV}$

$1.914 E+01 \mathrm{KEV}$

1.490E+01 KEV

$1.161 E+01 \mathrm{KEV}$

9.040E+00 KEV

$7.040 E+00 \mathrm{KEV}$

$5.483 E+00 \mathrm{KEV}$

4.270E+OO KEV

3.326E+00 KEV

2. $590 \mathrm{E}+00 \mathrm{KFV}$

$2.017 \mathrm{E}+00 \mathrm{KEV}$

$1.57 \mathrm{lE}+00 \mathrm{KEV}$

1. $223 E+U C K E V$

$7.52 B E-01 \mathrm{KEV}$

7.420E-01 KEV

5.779E-01 KEV

4.501E-O1 KEV

3.505E-O1 KEV

$2.730 \mathrm{E}-01 \mathrm{KEV}$

$2.126 \mathrm{E}-01 \mathrm{KEV}$

$1.656 \mathrm{E}-01 \mathrm{KEV}$

1.289E-OL KEV

1. $004 \mathrm{E}-01 \mathrm{KFV}$

$7.821 E-02 \mathrm{KEV}$

$0.091 E-02 \mathrm{KEV}$

4. $744 \mathrm{E}-02 \mathrm{KEV}$

$3.694 E-02$ KEV

$2.877 E-02$ KEV

$2.241 E+0 I$ IV

1.745E+OI EV

$1.359 E+01 \quad E V$

$1.058 E+01$ EV

$8.243 E+00$ EV

$6.420 E+00 \mathrm{EV}$

$5.000 E+00$ EV

3. B94E+00 EV

$3.033[+0 D \quad E V$

$2.362 \mathrm{~F}+00 \mathrm{FV}$

1. $839 E+00$ EV

$1.432 E+00 \quad E V$

$1.116 E+00 \quad E V$

8.688E-OI EV

$6.767 E-01$ IVV

$5.270 E-01$ EV

4.104E-01 EV

$3.196 E-01$ CV

$2.489 E-01 \mathrm{EV}$

$1.939 E-O L$ EV

$1.510 E-01$ EV

$1.176 \mathrm{E}-01 \mathrm{EV}$ 


\begin{tabular}{|c|c|c|c|c|c|c|c|c|c|c|c|c|c|c|c|c|}
\hline 0 & $\boldsymbol{\Omega}$ & 0 & 0 & 0 & 0 & 0 & 0 & 0 & 0 & 0 & 0 & 0 & 0 & $\boldsymbol{0}$ & 0 & 0 \\
\hline$\underset{\sim}{N}$ & $\stackrel{m}{m}$ & $\begin{array}{l}m \\
\infty \\
O \\
m\end{array}$ & $\begin{array}{l}0 \\
0 \\
0\end{array}$ & $\underset{m}{N}$ & $\stackrel{n}{R}$ & $\underset{n}{\tilde{n}}$ & $\begin{array}{l}m \\
0 \\
0 \\
g\end{array}$ & $\begin{array}{l}\boldsymbol{v} \\
0 \\
\mathfrak{v}\end{array}$ & $\underset{n}{\infty}$ & $\frac{\pi}{n}$ & $\underset{n}{m}$ & $\underset{n}{\rightarrow}$ & $\underset{m}{\vec{n}}$ & $\stackrel{4}{r}$ & $\underset{m}{m}$ & $\underset{\infty}{\infty}$ \\
\hline
\end{tabular}

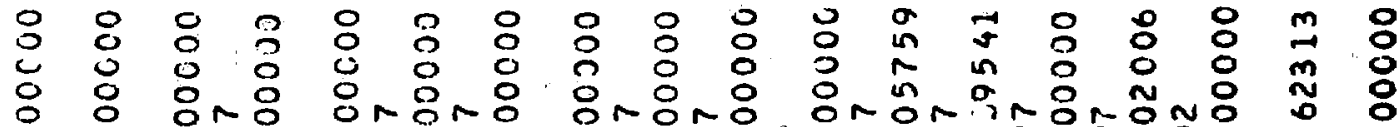

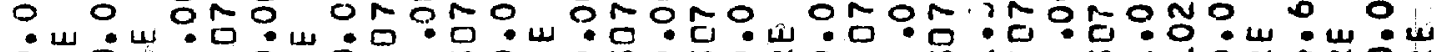

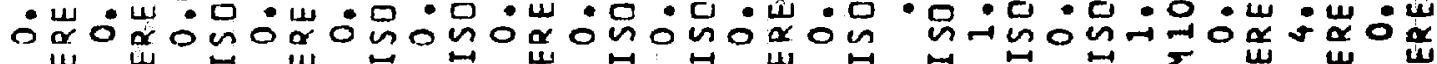

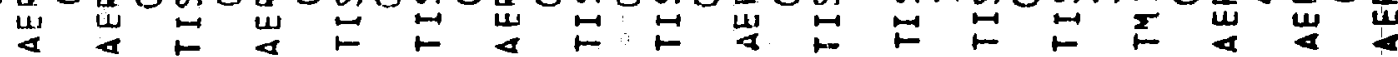

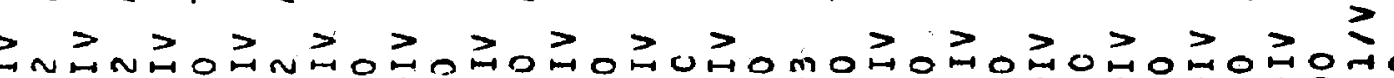

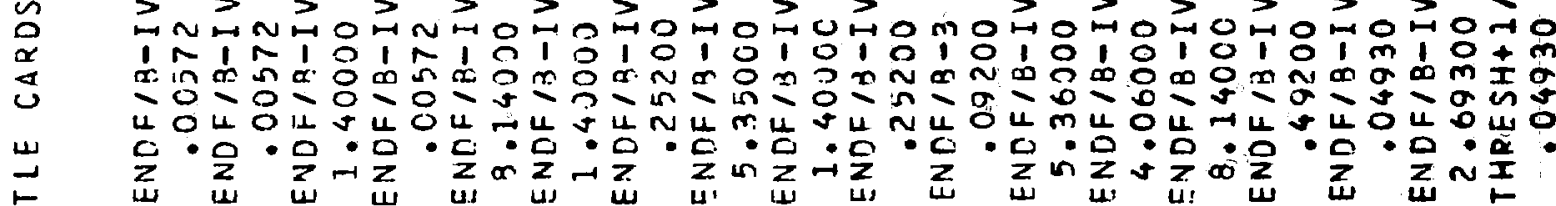

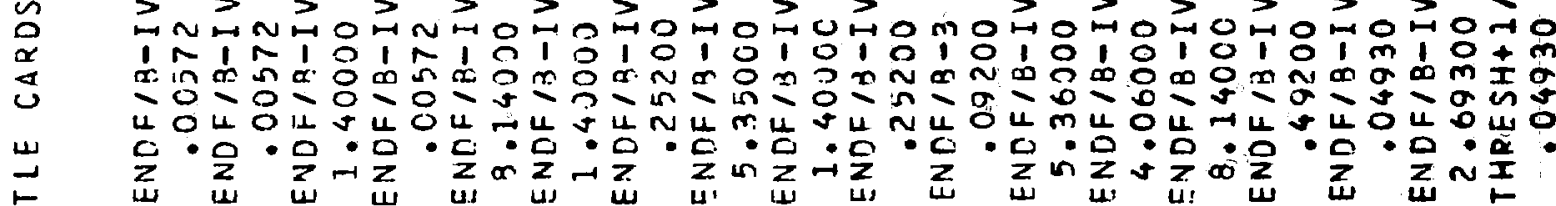

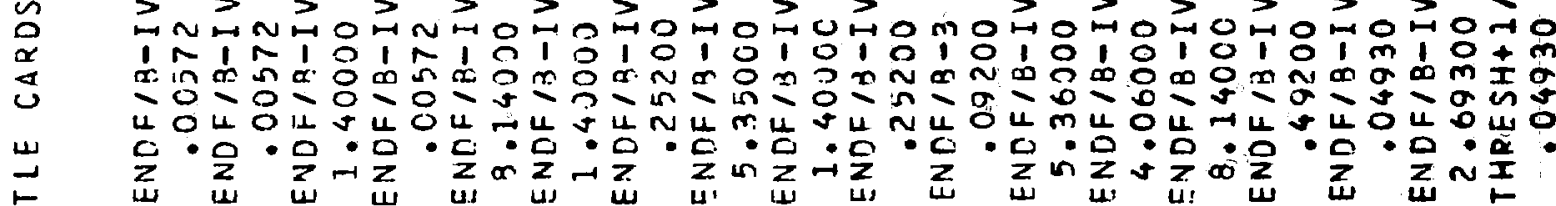

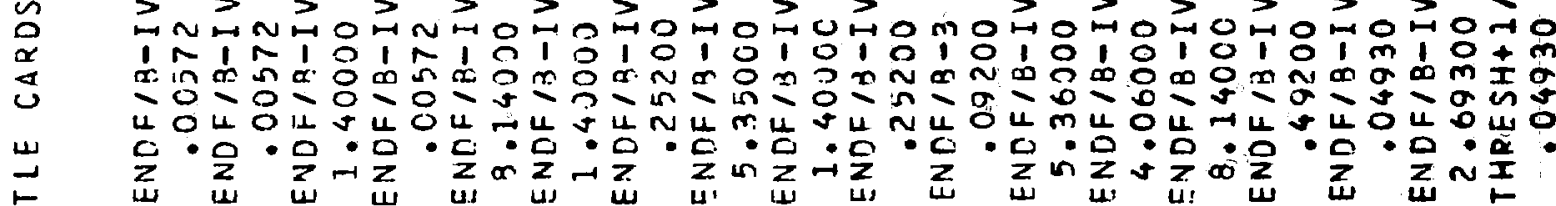

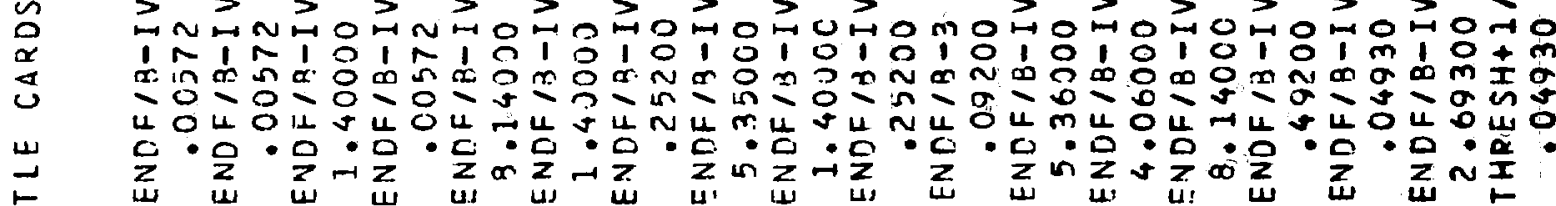

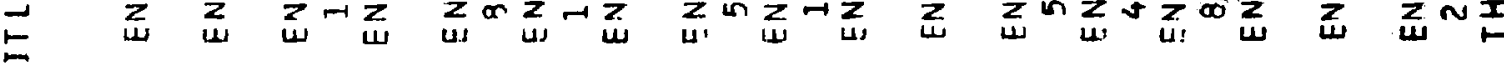

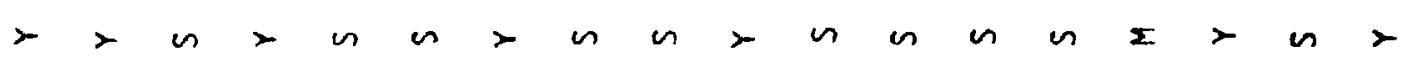

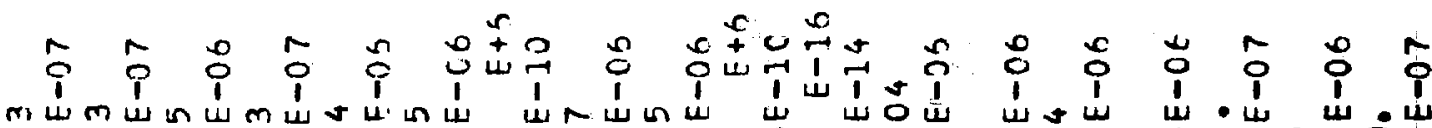

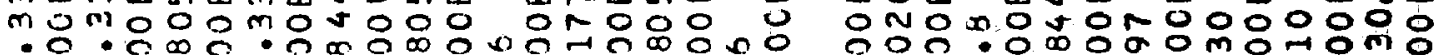

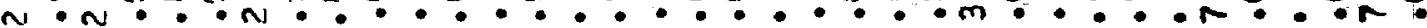

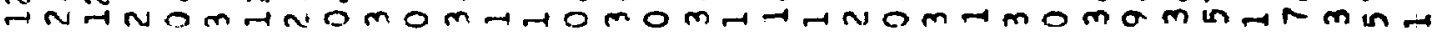

m

I I I I II I

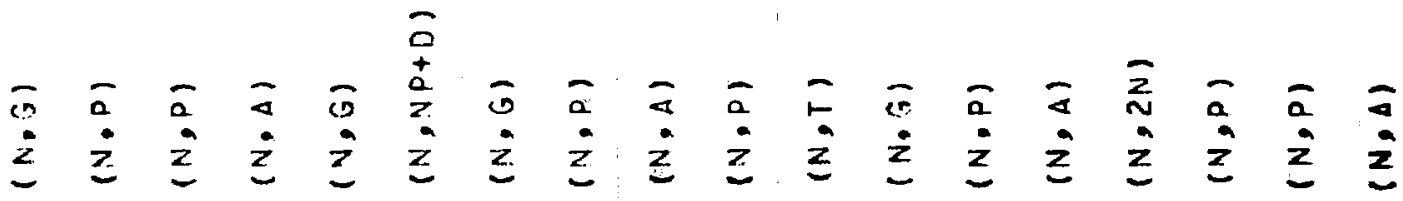

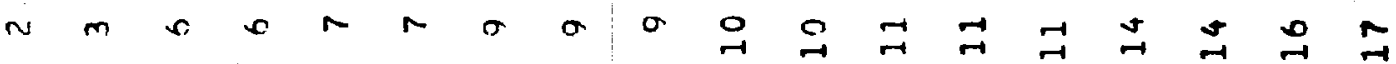

$$
\begin{aligned}
& \text { I }
\end{aligned}
$$




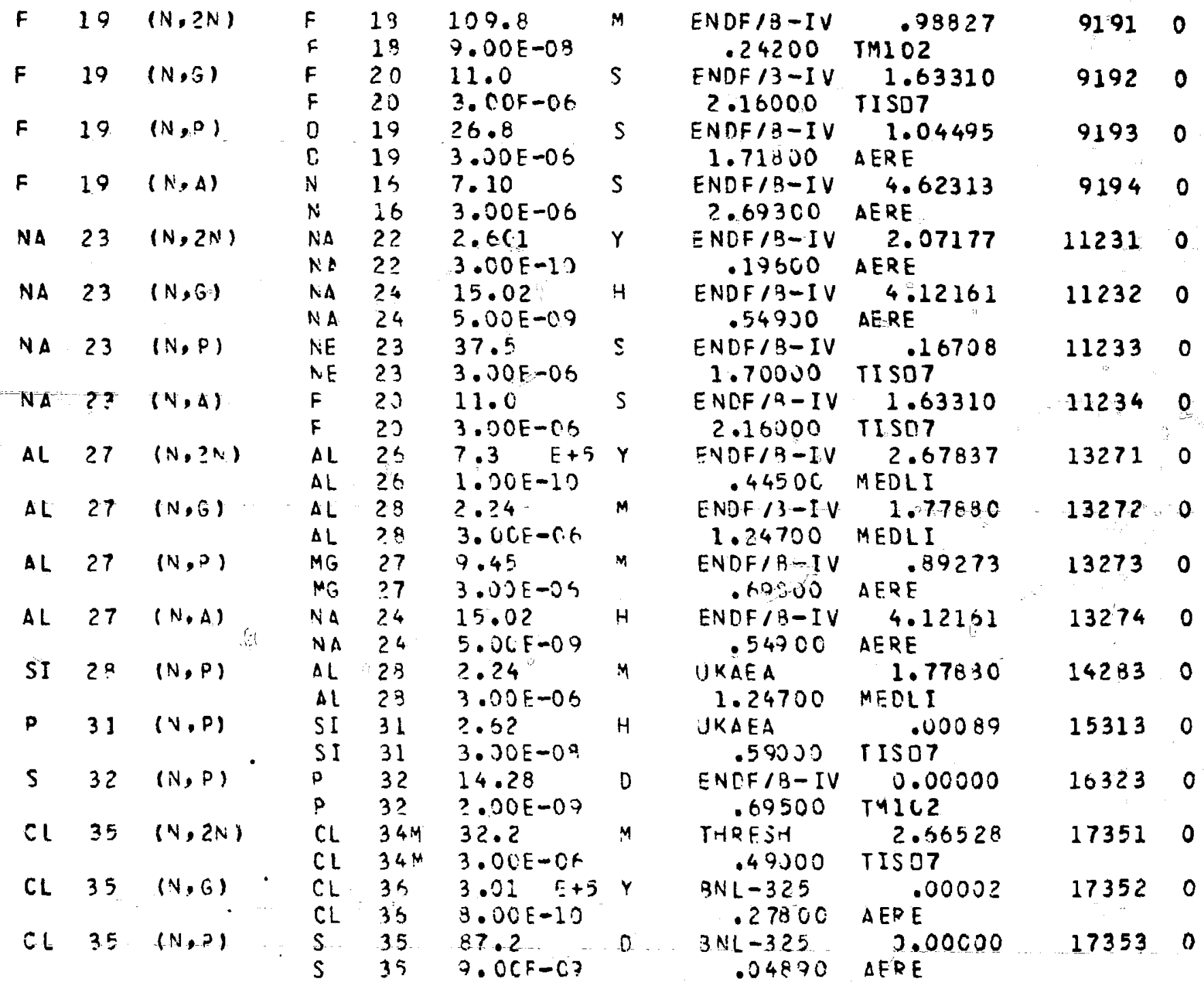




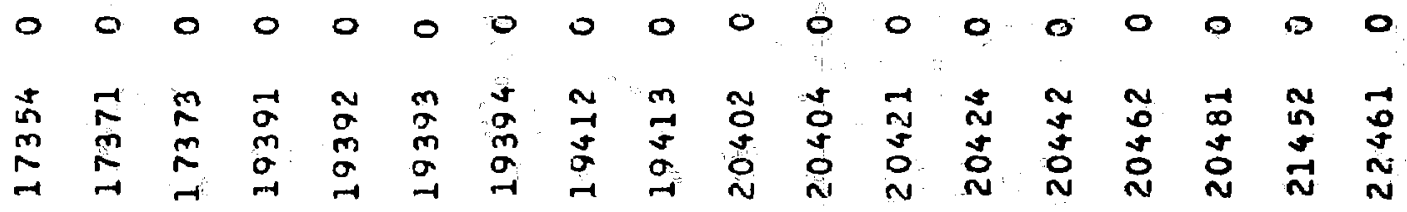

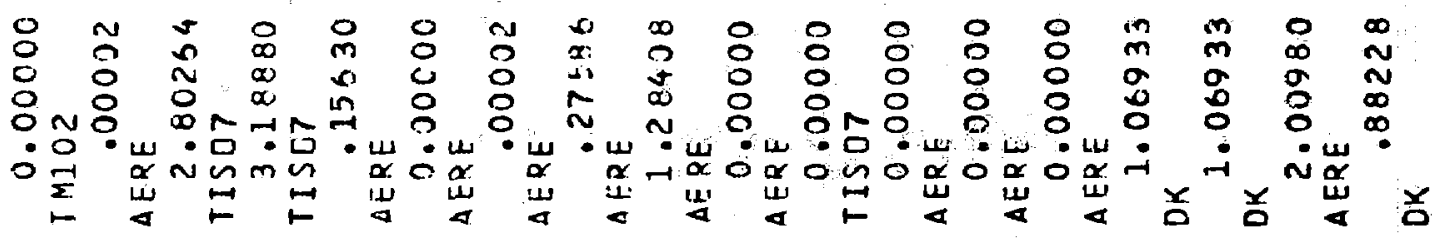

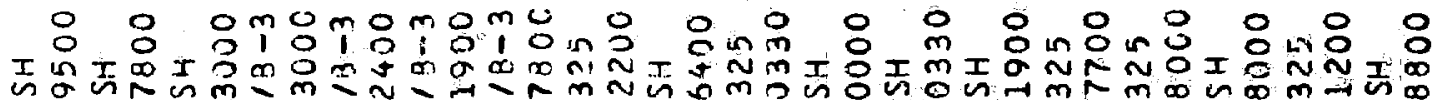

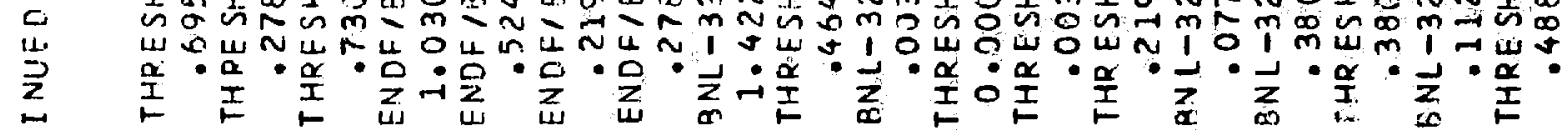

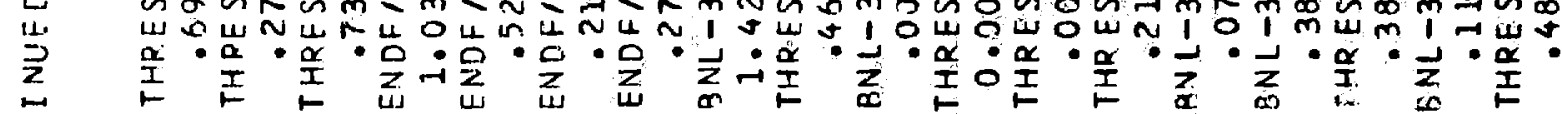

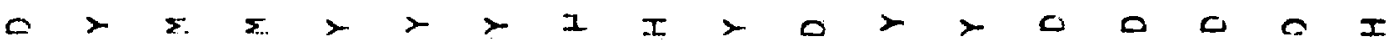

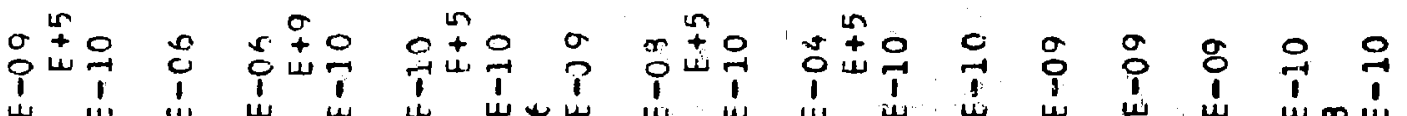
出

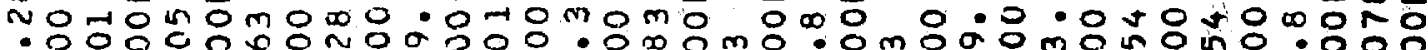

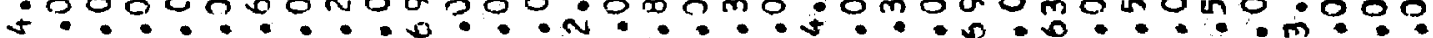

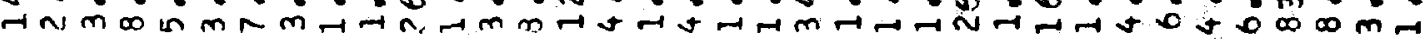

nincoram mm mm mmm $m$ m

п .

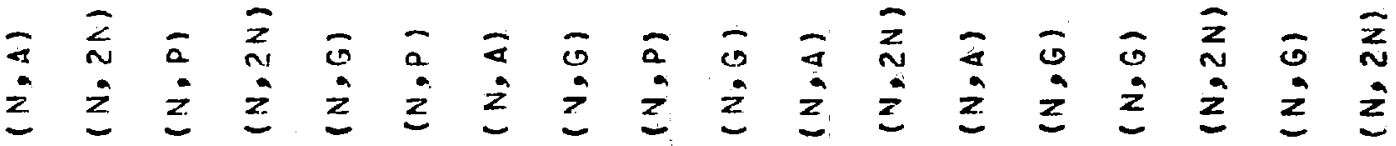

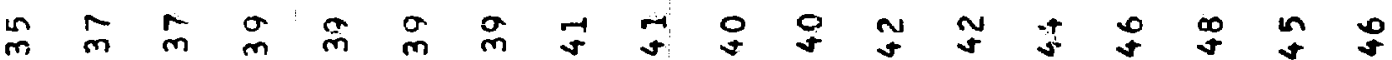

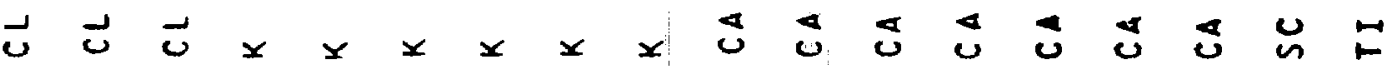




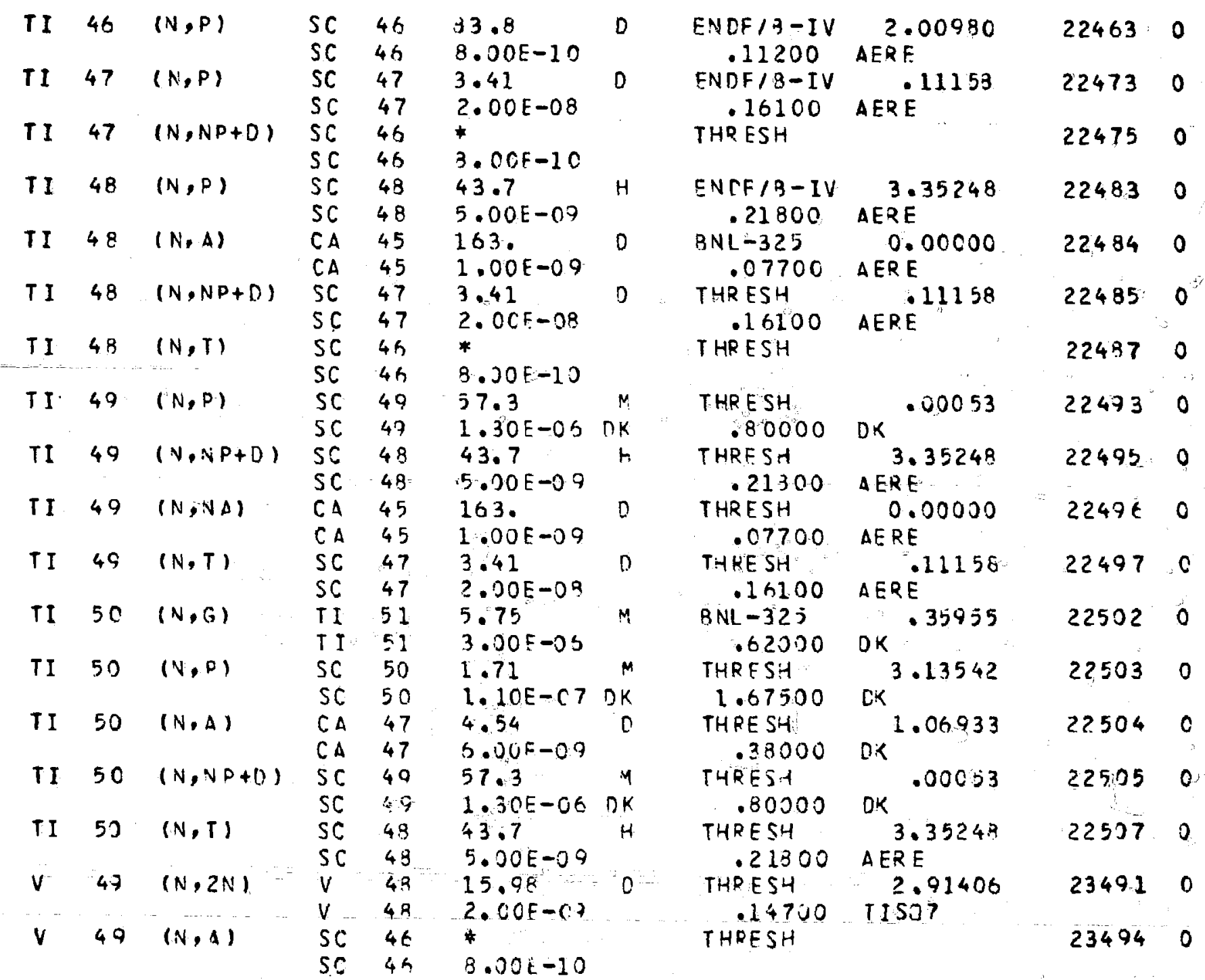




\begin{tabular}{|c|c|c|c|c|c|c|c|c|c|c|c|c|c|c|c|c|c|}
\hline 0 & 0 & 0 & 0 & $n$ & 0 & 0 & $\begin{array}{c}0 \\
0\end{array}$ & 0 & 0 & 0 & 0 & 0 & 0 & 0 & & 0 & 0 \\
\hline $\begin{array}{r}-1 \\
0 \\
\text { in }\end{array}$ & $\begin{array}{l}5 \\
0 \\
m\end{array}$ & $\begin{array}{l}0 \\
0 \\
m\end{array}$ & $\begin{array}{l}\sim \\
\vec{m}\end{array}$ & $\underset{m}{m}$ & $\underset{m}{n}$ & $\underset{m}{0}$ & $\overrightarrow{0}$ & $\underset{\text { in }}{N}$ & $\mathfrak{O}_{0}^{m}$ & $\begin{array}{l}\text { In } \\
\text { in }\end{array}$ & n & 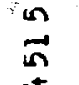 & $\begin{array}{l}\text { n' } \\
\text { in }\end{array}$ & $\begin{array}{l}m \\
n \\
n\end{array}$ & $=$ & $\stackrel{n}{n}$ & $m$ \\
\hline n & $N$ & $N$ & $N$ & N & $N$ & N & $\sim$ & $N$ & $N$ & $N$ & $\sim$ & $N^{n}$ & $N$ & $N$ & & $N$ & $N$ \\
\hline
\end{tabular}

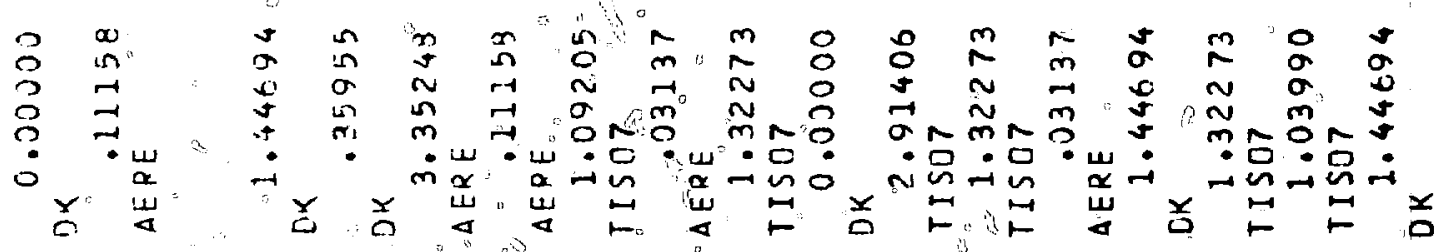

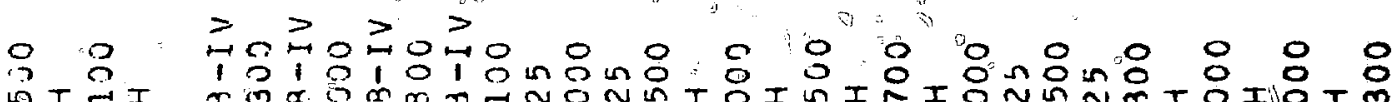

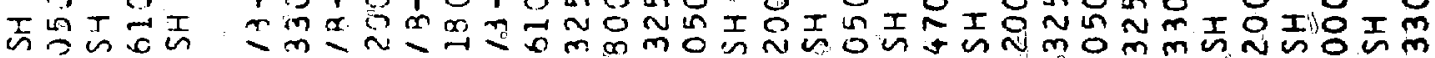

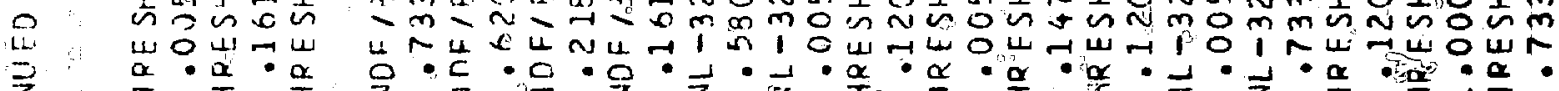

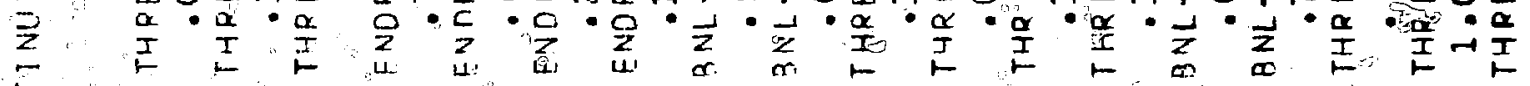

Tor

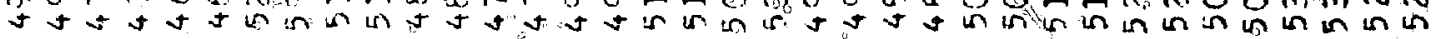

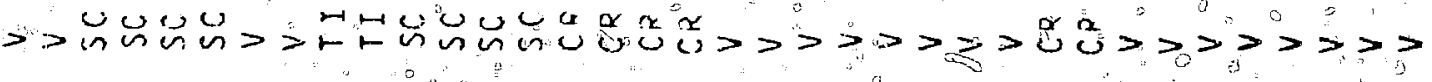

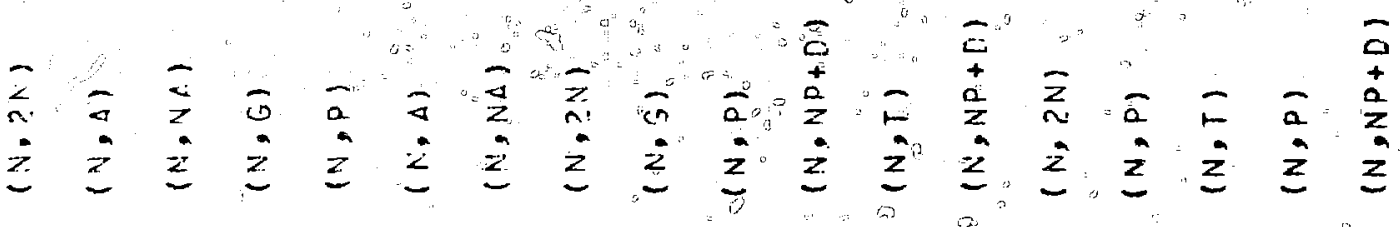

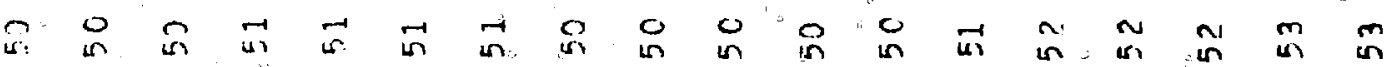

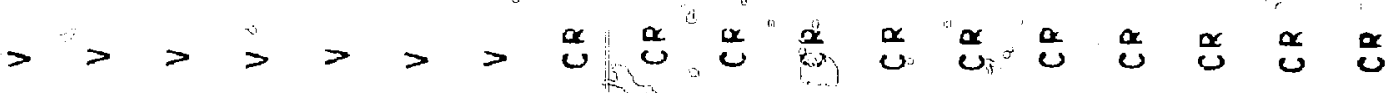




\begin{tabular}{|c|c|c|c|c|c|c|c|c|c|}
\hline$C R$ & 54 & $(N, D)$ & $\begin{array}{l}v \\
v\end{array}$ & $\begin{array}{l}54 \\
54\end{array}$ & $\begin{array}{l}43 . \\
3.00 F-06\end{array}$ & 5 & $\begin{array}{l}\text { THRE S4 } \\
=B 8000\end{array}$ & $\begin{array}{l}4.0,4000 \\
T I S 07\end{array}$ & 24543 \\
\hline$C R$ & 54 & $(N, \Delta)$ & $\begin{array}{l}\text { TI } \\
\text { TI }\end{array}$ & $\begin{array}{l}51 \\
51\end{array}$ & $\begin{array}{l}5.75 \\
3.00 E-08\end{array}$ & M & $\begin{array}{l}\text { THRESH } \\
=.62000\end{array}$ & DK .35955 & 24544 \\
\hline$C R$ & 54 & $(N, N P+D)$ & $\begin{array}{l}v \\
v\end{array}$ & $\begin{array}{l}53 \\
53\end{array}$ & $\begin{array}{l}1.55 \\
3.00 \mathrm{E}-0 \mathrm{~K}\end{array}$ & M & $\begin{array}{l}\text { THPESH } \\
1.00000\end{array}$ & $\begin{array}{l}1.03990 \\
\text { TISB7 }\end{array}$ & 24545 \\
\hline$C R=$ & 5.4 & $(N, T)$ & $\begin{array}{l}v \\
v\end{array}$ & $\begin{array}{l}52 \\
52\end{array}$ & $\begin{array}{l}3.76 \\
3.50 F-C 7 D\end{array}$ & $\begin{array}{c}M \\
D K\end{array}$ & $\begin{array}{c}\text { THP ESH } \\
.73300\end{array}$ & $\mathrm{OK}^{1.44 \in 94}$ & $\begin{array}{c}2.4547 \\
9\end{array}$ \\
\hline$M N$ & 53 & $(N, \geq N)$ & $\begin{array}{l}M N \\
M N\end{array}$ & $\begin{array}{l}52 \\
52\end{array}$ & $5.20 E-09$ & & THR ESH & & 25531 \\
\hline MN & 53 & $(N, A)$ & $\begin{array}{l}v^{\prime \prime} \\
v^{\prime \prime}\end{array}$ & $\begin{array}{l}50 \\
50\end{array}$ & $\begin{array}{l}\text { 4. } E+16 \\
1.00 E-10\end{array}$ & $Y$ & $\begin{array}{l}\text { THRESH } \\
\quad 12000\end{array}$ & TISO7 & 25534 \\
\hline$M N$ & 53 & $(N, N \Delta)$ & $\begin{array}{l}v \\
V\end{array}$ & $\begin{array}{l}49 \\
47\end{array}$ & $\begin{array}{l}331 . \\
? .50 E-07 \quad n\end{array}$ & $\begin{array}{c}D \\
n k\end{array}$ & $\begin{array}{r}\text { THRESH } \\
.00500\end{array}$ & $D x^{0.00000}$ & $25530^{\circ}$ \\
\hline$M N$ & 54 & $(N, 2 N)$ & $\begin{array}{l}M N \\
M N\end{array}$ & $\begin{array}{l}53 \\
53\end{array}$ & $\begin{array}{l}3.8 \quad E+6 \\
1.00 E-10\end{array}$ & $Y$ & $\begin{array}{l}\text { THRESH } \\
0.00000\end{array}$ & $\begin{array}{l}0.00000 \\
\text { TISO7 }\end{array}$ & 25541 \\
\hline$M N$ & 54 & $(N, N A)$ & $\begin{array}{l}v \\
v\end{array}$ & $\begin{array}{l}50 \\
50\end{array}$ & $\begin{array}{l}\text { 4. } E+16 \\
1.00 E=10\end{array}$ & $Y$ & $\begin{array}{l}\text { THRE SH } \\
.12000\end{array}$ & $\begin{array}{l}1.32273 \\
T[S 07\end{array}$ & 25546 \\
\hline$M N$ & 55 & $(N, 2 N)$ & $\begin{array}{l}M N \\
M N\end{array}$ & $\begin{array}{l}54 \\
54\end{array}$ & $\begin{array}{l}312.5 \\
1.30 E-09\end{array}$ & D & $\begin{array}{c}\text { FNDF/B-IV } \\
.00560\end{array}$ & $\triangle E Q E^{83463}$ & 25551 \\
\hline$M N$ & 55 & $(N, G)=$ & $\begin{array}{l}M N \\
M N\end{array}$ & $\begin{array}{l}55 \\
55\end{array}$ & $\begin{array}{l}? .580 \\
2.00 E-00\end{array}$ & $H$ & $\begin{array}{c}\text { ENDF / B - IV } \\
.80303\end{array}$ & $\begin{array}{l}1.78233 \\
\text { AERE }\end{array}$ & 25552 \\
\hline$M N$ & 55 & $(N, P)$ & $\begin{array}{l}C R \\
C R\end{array}$ & $\begin{array}{l}55 \\
55\end{array}$ & $\begin{array}{l}3.55 \\
3.00 \mathrm{E}-0.5\end{array}$ & M & $\begin{array}{c}\text { ENDF/B-IV } \\
1.00000\end{array}$ & $\operatorname{TISO07}$ & 25553 \\
\hline$M N$ & 55 & $(N, A)$ & $\begin{array}{l}v \\
v\end{array}$ & $\begin{array}{l}52 \\
5 ?\end{array}$ & $\begin{array}{l}3.76 \\
3.50 E-070\end{array}$ & M & $\begin{array}{c}\text { ENCF / B - IV } \\
.73300\end{array}$ & $c K^{1.44694}$ & $2555 \%$ \\
\hline FE & 54 & $(N, \geq N)$ & $\begin{array}{l}F E \\
F E\end{array}$ & $\begin{array}{l}53 \\
53\end{array}$ & 3. OCE-OS & & THRESH & & 26541 \\
\hline$F E$ & 54 & $(N, P)$ & $\begin{array}{l}M N \\
M N\end{array}$ & $\begin{array}{l}54 \\
54\end{array}$ & $\begin{array}{l}312.5 \\
1.0 C E-0.0\end{array}$ & 0 & $\begin{array}{c}\text { ENDF } 13-\text { IV } \\
.00550\end{array}$ & $\triangle E R E^{93453^{\circ}}$ & $2 \in 543$ \\
\hline FE & 54 & $(N, A)$ & $\begin{array}{l}C R \\
C Q\end{array}$ & $\begin{array}{l}51 \\
51\end{array}$ & $\begin{array}{l}27.71 \\
8.007-09\end{array}$ & c & $\begin{array}{r}P N L=325 \\
.00500\end{array}$ & $A E R E$ & 26544 \\
\hline FE & 54 & $(N, N P+O)$ & $M N$ & $\begin{array}{l}53 \\
53\end{array}$ & $\begin{array}{l}3.3 \text { E }+6 \\
1.00 E-10\end{array}$ & $r$ & $\begin{array}{l}\text { THRESH } \\
0.0000 \mathrm{C}\end{array}$ & $\begin{array}{l}0.00030 \\
\text { TIS07 }\end{array}$ & 26545 \\
\hline 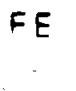 & 54 & $(N, T)$ & $M N$ & $5 ?$ & $5.00 E-39$ & & THRESH & & 26547 \\
\hline
\end{tabular}




\begin{tabular}{|c|c|c|c|c|c|c|c|c|c|}
\hline FE & 55 & $(N, N P+D)$ & $\begin{array}{l}\text { MN } \\
M N\end{array}$ & $\begin{array}{l}54 \\
54\end{array}$ & $\begin{array}{l}312.5 \\
1.00 E-09\end{array}$ & D & $\begin{array}{l}\text { TARFSH } \\
.00550\end{array}$ & $\triangle E R \dot{E}^{.83463}$ & 26555 \\
\hline F E & 55 & $(N, N A)$ & $\begin{array}{l}\text { CR } \\
C Q\end{array}$ & $\begin{array}{l}51 \\
51\end{array}$ & $\begin{array}{l}27.71 \\
\text { B. CCE-CB }\end{array}$ & 0 & $\begin{array}{l}\text { THPESH } \\
.00500\end{array}$ & $\triangle .^{03137}$ & $2+556$ \\
\hline FE & 56 & $(N, 2 N)$ & $\begin{array}{l}F E \\
F E\end{array}$ & $\begin{array}{l}55 \\
55\end{array}$ & $\begin{array}{l}2.7 \\
3.30 E-03\end{array}$ & $Y$ & $\begin{array}{l}\text { THRESH } \\
.00590\end{array}$ & $\begin{array}{l}0.00000 \\
\text { AERE }\end{array}$ & 26561 \\
\hline FE & 56 & $(A, P)$ & $\begin{array}{l}M N \\
M N\end{array}$ & $\begin{array}{l}56 \\
56\end{array}$ & $\begin{array}{l}2.580 \\
2.00 E-08\end{array}$ & 4 & $\begin{array}{c}\text { ENDF/B-IV } \\
.80000\end{array}$ & $\begin{array}{l}\text { 1. } 78233 \\
\triangle E R E\end{array}$ & 26553 \\
\hline$F E$ & 55 & $(N, T)$ & $\begin{array}{l}M N \\
M N\end{array}$ & $\begin{array}{l}54 \\
54\end{array}$ & $\begin{array}{l}312.5 \\
1.0 C F-C 9\end{array}$ & 0 & $\begin{array}{l}\text { THR ESH } \\
.00500\end{array}$ & $\triangle E R E^{.83463}$ & 26567 \\
\hline FE & 57 & $(N, P)$ & $\begin{array}{l}M N \\
M N\end{array}$ & $\begin{array}{l}57 \\
57\end{array}$ & $\begin{array}{l}1.59 \\
3.00 E-05\end{array}$ & $M$ & $\begin{array}{l}\text { THPESH } \\
.97000\end{array}$ & TIS:07 & 26573 \\
\hline FE & 57 & $(N, N P+D)$ & $\begin{array}{l}M N \\
M N\end{array}$ & $\begin{array}{l}56 \\
56\end{array}$ & $\begin{array}{l}2.58 C \\
2.00 E-08\end{array}$ & 4 & $\begin{array}{l}\text { THRESH } \\
.80000\end{array}$ & $\begin{array}{l}1.78233 \\
\triangle E R E\end{array}$ & 26575 \\
\hline FE & 58 & $(N, G)$ & $\begin{array}{l}F E \\
F E\end{array}$ & $\begin{array}{l}59 \\
59\end{array}$ & $\begin{array}{l}44.6 \\
2.00 E-09\end{array}$ & $\mathrm{D}$ & $\begin{array}{c}\text { ENDF } / 8-\text { IV } \\
.11800\end{array}$ & $\begin{array}{l}1.19234 \\
\triangle E R E\end{array}$ & 26582 \\
\hline FE & 59 & $(N, P)$ & $M N$ & $\begin{array}{l}58 \\
53\end{array}$ & $35.00 E-06$ & $S$ & $\begin{array}{l}\text { THRESH } \\
1.37000\end{array}$ & $\begin{array}{l}2.53675 \\
\text { TIS } 07\end{array}$ & 26583 \\
\hline FE & 58 & $(N, A)$ & $\begin{array}{l}C R \\
C R\end{array}$ & $\begin{array}{l}55 \\
55\end{array}$ & $\begin{array}{l}3.55 \\
3.00 E-O S\end{array}$ & $M$ & $\begin{array}{l}\text { THRESH } \\
1.00000\end{array}$ & TIS 007 & 26584 \\
\hline F E & 58 & $(N, N D+D)$ & $\begin{array}{l}M N \\
M N\end{array}$ & $\begin{array}{l}57 \\
57\end{array}$ & $\begin{array}{l}1.59 \\
3.00 E-06\end{array}$ & $M$ & $\begin{array}{l}\text { THR ESH } \\
.97000\end{array}$ & TISO7 & 26585 \\
\hline FE & 58 & $(N, T)$ & $\begin{array}{l}M N \\
M N\end{array}$ & $\begin{array}{l}56 \\
56\end{array}$ & $\begin{array}{l}2.580 \\
2.00 E-08\end{array}$ & $H$ & $\begin{array}{l}\text { THRESH } \\
.80000\end{array}$ & $\begin{array}{l}1.78233 \\
\triangle E R E\end{array}$ & 26587 \\
\hline CD & 57 & $(N, 2 N)$ & $\begin{array}{l}\mathrm{CO} \\
\mathrm{CO}\end{array}$ & $\begin{array}{l}56 \\
56\end{array}$ & $\begin{array}{l}78.5 \\
1.00 E-10\end{array}$ & D & $\begin{array}{r}\text { THR E SH } \\
.11000\end{array}$ & $\begin{array}{l}3.64227 \\
\text { T IS } 67\end{array}$ & 27571 \\
\hline $\mathrm{CO}$ & 57 & $(N, A)$ & $\begin{array}{l}M N \\
M N\end{array}$ & $\begin{array}{l}54 \\
54\end{array}$ & $\begin{array}{l}312.5 \\
1.00 E-09\end{array}$ & $D$ & $\begin{array}{l}\text { THRESH } \\
.00560\end{array}$ & $\triangle E Q \dot{E}^{.83463}$ & 27574 \\
\hline $\mathrm{CO}$ & 57 & $(N, N A)$ & $\begin{array}{l}M N \\
M N\end{array}$ & $\begin{array}{l}53 \\
53\end{array}$ & $\begin{array}{ll}3.8 & E+6 \\
1.00 E-10\end{array}$ & $Y$ & $\begin{array}{l}\text { THRESH } \\
0.00000\end{array}$ & $\begin{array}{l}0.00000 \\
\text { TIS } 07\end{array}$ & 27576 \\
\hline $\mathrm{CO}$ & 59 & $(N, 2 N)$ & $\begin{array}{l}\mathrm{CO} \\
\mathrm{CO}\end{array}$ & $\begin{array}{l}58 \\
58\end{array}$ & $\begin{array}{l}70.8 \\
2.00 E-09\end{array}$ & $D$ & $\begin{array}{c}\text { ENDF / B- IV } \\
.03650\end{array}$ & $A E R:^{.97420}$ & 27591 \\
\hline $\mathrm{CO}$ & 59 & $(N, G)$ & $\begin{array}{l}\mathrm{CO} \\
\mathrm{CO}\end{array}$ & $\begin{array}{l}60 \\
60\end{array}$ & $\begin{array}{l}5.27 \\
3.00 E-10\end{array}$ & $Y$ & $\begin{array}{c}\text { ENDF/B-IV } \\
.09670\end{array}$ & $\begin{array}{l}2.50443 \\
\text { AERE }\end{array}$ & 27592 \\
\hline$C O$ & 59 & $(N, P)$ & $\begin{array}{l}F E \\
F E\end{array}$ & $\begin{array}{l}59 \\
59\end{array}$ & $\begin{array}{l}44.6 \\
2.00 E-09\end{array}$ & D & $\begin{array}{c}\text { ENDF/B-IV } \\
.11900\end{array}$ & $\begin{array}{l}1.19234 \\
\triangle E R E\end{array}$ & 27593 \\
\hline
\end{tabular}


TABLE II - CONTINUED

\begin{tabular}{|c|c|c|c|c|c|c|c|c|c|c|}
\hline CD & 59 & $(N, A)$ & $\begin{array}{l}M N \\
M N\end{array}$ & $\begin{array}{l}55 \\
56\end{array}$ & $\begin{array}{l}2.580 \\
2.00 E-0 F\end{array}$ & $\mu$ & $\begin{array}{c}\text { FNDF / B-IV } \\
.80000\end{array}$ & $\begin{array}{l}1.79233 \\
\text { AERE }\end{array}$ & 27594 & 0 \\
\hline $\mathrm{CO}$ & 60 & $(N, P)$ & $\begin{array}{l}F E \\
F E\end{array}$ & $\begin{array}{l}60 \\
60\end{array}$ & $\begin{array}{l}\text { 1. } \quad E+5 \\
1.00 E-10\end{array}$ & Y & $\begin{array}{l}\text { THR ESH } \\
.05000\end{array}$ & $\begin{array}{l}0.00000 \\
\text { TIS } 07\end{array}$ & 27603 & 0 \\
\hline $\mathrm{CO}$ & 60 & $(N, \Delta)$ & $\begin{array}{l}M N \\
M N\end{array}$ & $\begin{array}{l}57 \\
57\end{array}$ & $\begin{array}{l}1.59 \\
3.00 E-O S\end{array}$ & $M$ & $\begin{array}{l}\text { THRE SH } \\
.97000\end{array}$ & TISO7 & 27604 & 0 \\
\hline $\mathrm{CO}$ & $6 C$ & $(V, N P+n)$ & $\begin{array}{l}F E \\
F E\end{array}$ & $\begin{array}{l}59 \\
59\end{array}$ & $\begin{array}{l}44.6 \\
2.00 E-C 9\end{array}$ & D & $\begin{array}{l}\text { THRESH } \\
.11800\end{array}$ & $\begin{array}{l}1.19234 \\
\triangle E R E\end{array}$ & 27505 & 0 \\
\hline $\mathrm{CO}$ & 60 & $(N, N \Delta)$ & $\begin{array}{l}M N \\
M N\end{array}$ & $\begin{array}{l}56 \\
56\end{array}$ & $\begin{array}{l}2.580 \\
2.00 E-03\end{array}$ & $H$ & $\begin{array}{l}\text { THRESH } \\
.80000\end{array}$ & $\begin{array}{l}1.78233 \\
A E R E\end{array}$ & 27606 & 0 \\
\hline NI & 58 & $(N, 2 N)$ & $\begin{array}{l}N I \\
M I\end{array}$ & $\begin{array}{l}57 \\
57\end{array}$ & $\begin{array}{l}36.1 \\
1.0 C F-10\end{array}$ & $H$ & $\begin{array}{c}\text { ENDF } 13-\text { IV } \\
.13000\end{array}$ & $\begin{array}{l}2.10649 \\
\text { TI SO } 7\end{array}$ & 28581 & 0 \\
\hline N I & 59 & $(N, G)$ & $\begin{array}{l}N I \\
N I\end{array}$ & $\begin{array}{l}59 \\
59\end{array}$ & $\begin{array}{l}3.05+4 \\
2.00 E-O R\end{array}$ & $Y$ & $\begin{array}{c}3 N L-3 ? 2 \\
.00700\end{array}$ & $\begin{array}{l}0.00000 \\
\triangle E R E\end{array}$ & 28582 & 0 \\
\hline $\mathrm{NI}$ & 58 & $(N, P)$ & $\begin{array}{l}C D \\
C O\end{array}$ & $\begin{array}{l}59 \\
59\end{array}$ & $\begin{array}{l}70.8 \\
2.00 E-09\end{array}$ & $D$ & $\begin{array}{c}\text { ENCF / R-IV } \\
.03650\end{array}$ & $\triangle E R \dot{E}^{97423}$ & 28583 & 0 \\
\hline N I & 58 & $(N, \Delta)$ & $\begin{array}{l}F E \\
F E\end{array}$ & $\begin{array}{l}55 \\
55\end{array}$ & $\begin{array}{l}2.7 \\
3.00 E-O Q\end{array}$ & $Y$ & $\begin{array}{l}\text { THRE 54 } \\
.00590\end{array}$ & $\triangle E R E$ & 28584 & 0 \\
\hline NI & 58 & $(N, N P+D)$ & $\begin{array}{ll}C \\
C 0\end{array}$ & $\begin{array}{l}57 \\
57\end{array}$ & $\begin{array}{l}271 . \\
3.00 E-09\end{array}$ & $c$ & $\begin{array}{l}\text { THFFS4 } \\
.02 ? 33\end{array}$ & $\triangle E R \dot{E}^{12148}$ & 25595 & 0 \\
\hline VI & 58 & $(N, T)$ & $\begin{array}{l}C 0 \\
C 0\end{array}$ & $\begin{array}{l}56 \\
56\end{array}$ & $\begin{array}{l}78.5 \\
1.00 E-10\end{array}$ & 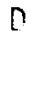 & $\begin{array}{l}\text { THRE SH } \\
.11000\end{array}$ & $\begin{array}{l}3.64227 \\
\text { TIS07 }\end{array}$ & 29537 & 0 \\
\hline N I & 57 & $(N, N P+n)$ & $\begin{array}{l}\mathrm{CO} \\
\mathrm{CO}\end{array}$ & $\begin{array}{l}53 \\
58\end{array}$ & $? .00 \mathrm{E}-\mathrm{Cg}$ & & THRFSH & & 28395 & $?$ \\
\hline NI & 59 & $(N, N A)$ & $\begin{array}{l}F E \\
F F\end{array}$ & $\begin{array}{l}55 \\
55\end{array}$ & $\begin{array}{l}2.7 \\
3.00 E-05\end{array}$ & $Y$ & $\begin{array}{l}\text { THRESH } \\
.00590\end{array}$ & $\begin{array}{l}0.00000 \\
\triangle E R E\end{array}$ & 28596 & 0 \\
\hline N I & 50 & $(N, 2 N)$ & $\begin{array}{l}N I \\
N I\end{array}$ & $\begin{array}{l}59 \\
50\end{array}$ & $\begin{array}{l}\text { 8. } \quad E+4 \\
2.00 E-C Q\end{array}$ & $Y$ & $\begin{array}{l}\text { THR ESH } \\
.00700\end{array}$ & $\begin{array}{l}0.00000 \\
\text { AERE }\end{array}$ & 29501 & 0 \\
\hline$N I$ & 60 & $(N, D)$ & $\begin{array}{l}C \square \\
0.0\end{array}$ & 60 & $\begin{array}{l}5 . ? 7 \\
3.0 J E-10\end{array}$ & $Y$ & $\begin{array}{c}\text { ENDF/B-IV } \\
.096 .70\end{array}$ & $\triangle \mathbb{R R E}^{2.5 C 443}$ & 28603 & 0 \\
\hline NI & 60 & $(N, T)$ & $\begin{array}{l}\mathrm{CD} \\
\mathrm{C}]\end{array}$ & $\begin{array}{l}54 \\
58\end{array}$ & $? .00 F-00$ & & THRES-1 & & 285.27 & 0 \\
\hline$N I$ & 61 & $(N, P)$ & $\begin{array}{ll}\mathrm{CO} \\
\mathrm{CO}\end{array}$ & $\begin{array}{l}61 \\
61\end{array}$ & $\begin{array}{l}1.65 C \\
7.50 E-C B\end{array}$ & $H$ & $\begin{array}{l}\text { THRFj4 } \\
.46020\end{array}$ & TISति & 28513 & 0 \\
\hline NI & 61 & $(N, N P+D)$ & $\begin{array}{l}\mathrm{CD} \\
\mathrm{CO}\end{array}$ & $\begin{array}{l}60 \\
50\end{array}$ & $3.00 E-10$ & & THRESH & & 28515 & 0 \\
\hline
\end{tabular}




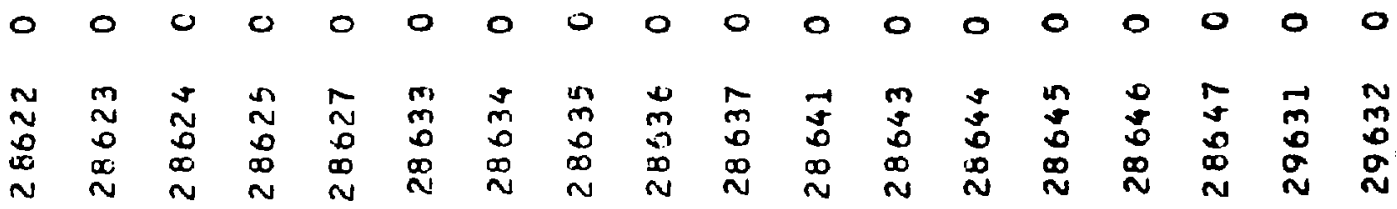

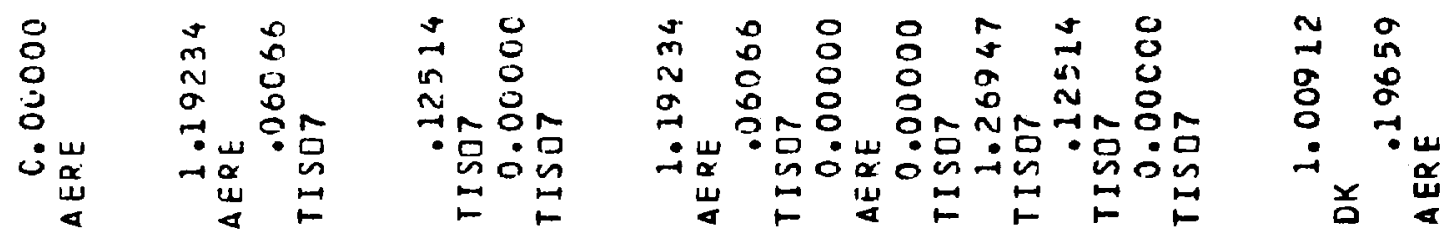

$\begin{array}{llllllllllllllllll}0 & 0 & 0 & 0 & 0 & 0 & 0 & 0 & 0 & 0 & 0 & 0 & 0 & 0\end{array}$

ñII I

10ш ш

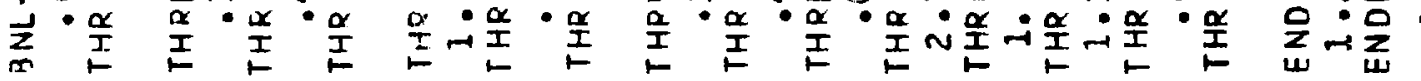

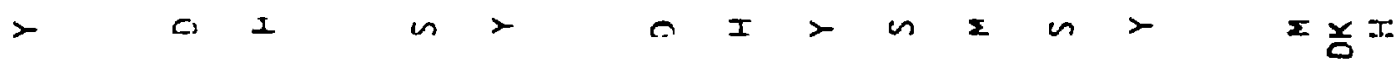

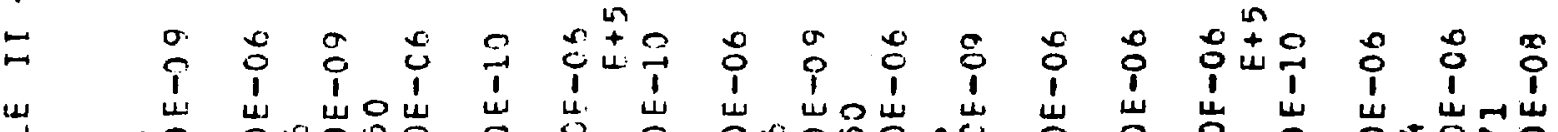

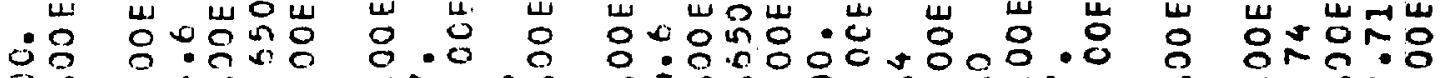
o? ? ? ? ? ? ? ? ? ? ? ? ?

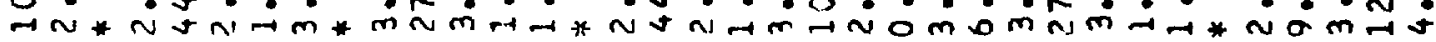

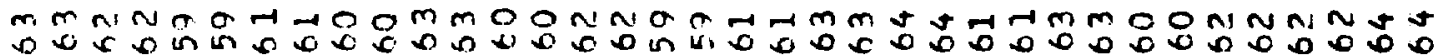

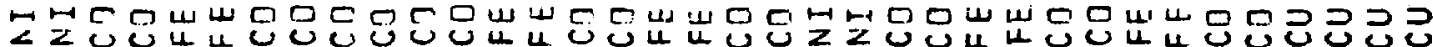

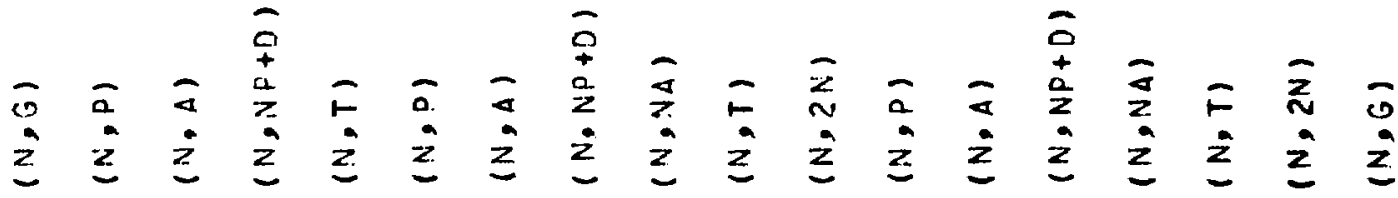

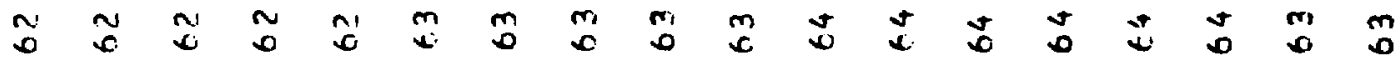

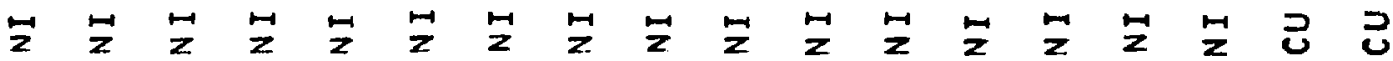




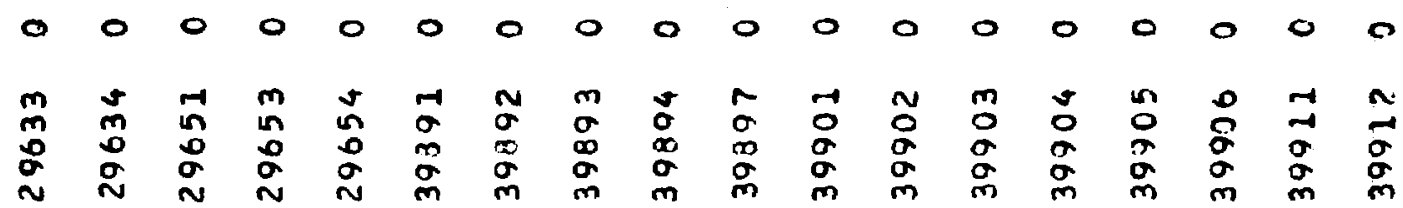

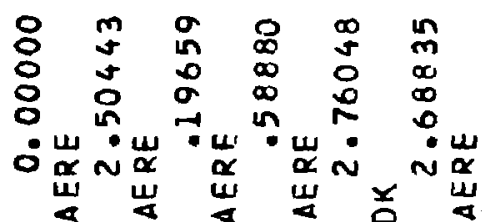

momo

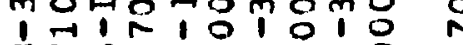

$a n m a n g m a n$

$40 \mu 0 \|, 4$ ㄴ.

$\sum_{u}^{0} \cdot \frac{0}{z} \cdot \frac{0}{z} \cdot \frac{0}{z} \cdot \frac{0}{z}+\frac{1}{u} \cdot 0$

$>I I I I 0$

$\overbrace{0}^{\infty}$

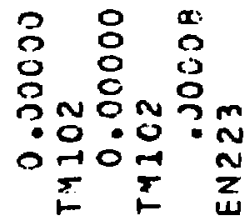

in

in

Ni

- 0

$\underset{1}{E}$

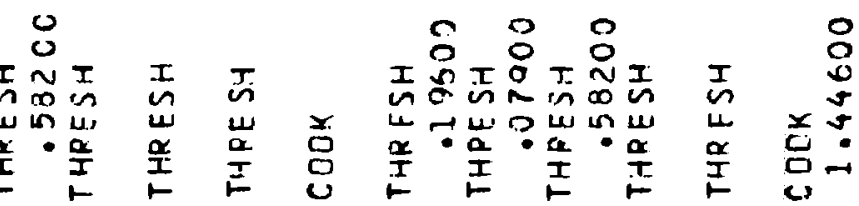

$>\quad \omega$

I

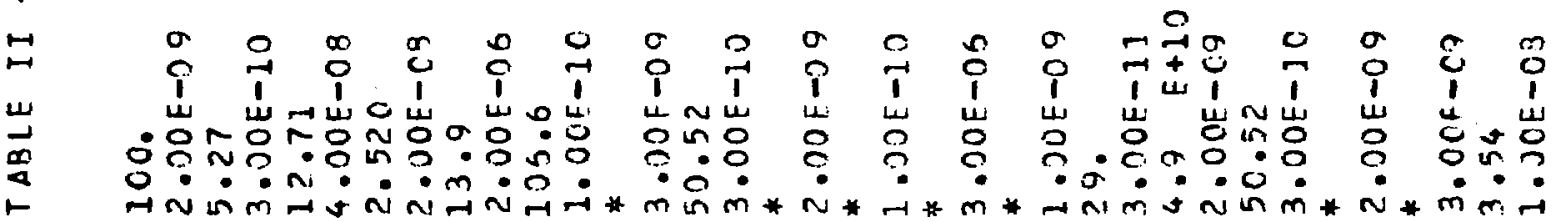

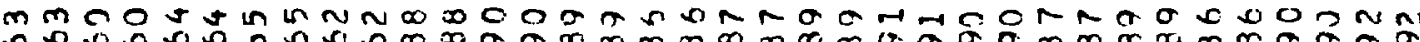

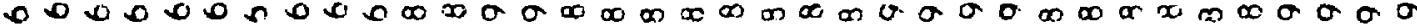

m

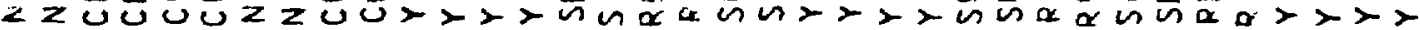

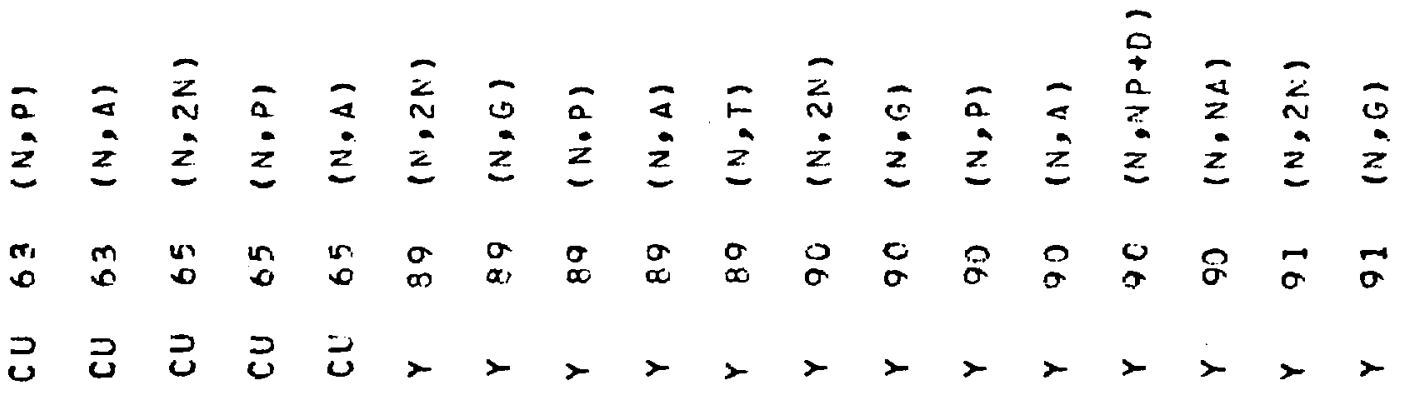




\begin{tabular}{|c|c|c|c|c|c|c|c|c|c|}
\hline$Y$ & 91 & $(N, P)$ & $\begin{array}{l}S R \\
S R\end{array}$ & $\begin{array}{l}91 \\
91\end{array}$ & $\begin{array}{l}9.52 \\
9.00 E-09\end{array}$ & $H$ & $\begin{array}{l}\text { THRE SH } \\
\text { E } E 5500\end{array}$ & $\begin{array}{l}1.02485 \\
T M 102\end{array}$ & 39713 \\
\hline$Y$ & 91 & $(N, \Delta)$ & $\begin{array}{l}\text { R B } \\
\text { R B }\end{array}$ & $\begin{array}{l}88 \\
39\end{array}$ & $\begin{array}{l}17.7 \\
3 . J C F-06\end{array}$ & M & $\begin{array}{l}\text { THRESH } \\
2.08100\end{array}$ & TM102 & 39914 \\
\hline$Y$ & 91 & $(N, N P+D)$ & $\begin{array}{l}S P \\
S R\end{array}$ & $\begin{array}{l}90 \\
9.3\end{array}$ & $\begin{array}{l}29 . \\
3.0 O E-11\end{array}$ & $Y$ & $\begin{array}{l}\text { THRESH } \\
.19600\end{array}$ & $\begin{array}{l}0.00000 \\
T 4102\end{array}$ & 39715 \\
\hline Y & 91 & $(N, N \Delta)$ & $\begin{array}{l}\text { PR } \\
\text { RB }\end{array}$ & $\begin{array}{l}87 \\
87\end{array}$ & $\begin{array}{l}4.9 \quad E+10 \\
2.00 E-09\end{array}$ & Y & $\begin{array}{l}\text { THR ESH } \\
.07700\end{array}$ & $\begin{array}{l}0.00000 \\
\text { TY102 }\end{array}$ & 39916 \\
\hline$Y$ & 91 & $(N, T)$ & $\begin{array}{l}S R \\
S R\end{array}$ & $\begin{array}{l}89 \\
89\end{array}$ & $\begin{array}{l}50.52 \\
3.0 \mathrm{CE}-10\end{array}$ & D & $\begin{array}{l}\text { THR ESH } \\
.58200\end{array}$ & EN2Z3 & 39917 \\
\hline$Z R$ & 90 & $(N, 2 N)$ & $\begin{array}{l}2 R \\
2 R\end{array}$ & $\begin{array}{l}97 \\
89\end{array}$ & $\begin{array}{l}78.5 \\
1.00 E-10\end{array}$ & $H$ & $\begin{array}{l}\text { UKAEA } \\
.08000\end{array}$ & $\begin{array}{l}1 \cdot 16581 \\
\text { TISD }\end{array}$ & 40901 \\
\hline$Z R$ & 90 & $(N, P)$ & $\begin{array}{l}Y \\
Y\end{array}$ & $\begin{array}{l}90 \\
90\end{array}$ & $3.00 E-09$ & & THRE SH & & 40903 \\
\hline$Z R$. & $9 \mathrm{C}$ & $(A, A)$ & $\begin{array}{l}S R \\
S R\end{array}$ & $\begin{array}{l}97 \\
87\end{array}$ & $1.00 E-10$ & & THRESH & & 40904 \\
\hline$Z R$ & 90 & $(N, N P+D)$ & $\begin{array}{l}Y \\
Y\end{array}$ & $\begin{array}{l}89 \\
89\end{array}$ & $3.00 E-0 S$ & & THRESH & & 40905 \\
\hline ZR & 90 & $(N, T)$ & $\begin{array}{l}Y \\
Y\end{array}$ & $\begin{array}{l}88 \\
88\end{array}$ & 1. $0 G E-10$ & & THR ESH & & 40907 \\
\hline ZR & 91 & $(N, 2 N)$ & $\begin{array}{l}Z R \\
Z R\end{array}$ & $\begin{array}{l}90 \\
90\end{array}$ & $3.00 E-C 6$ & & THRESH & & 40911 \\
\hline$Z R$ & 91 & $(N, P)$ & $\begin{array}{l}y \\
y\end{array}$ & 91 & * $1.00 E-09$ & & THR ESH & & 40913 \\
\hline ZR & 91 & $(N, N P+D)$ & $\begin{array}{l}Y \\
Y\end{array}$ & $\begin{array}{l}90 \\
93\end{array}$ & $3.00 E-09$ & & THR ESH & & 40915 \\
\hline ZR & 91 & $(N, N \Delta)$ & $\begin{array}{l}S R \\
S R\end{array}$ & $\begin{array}{l}87 \\
87\end{array}$ & * $1 \cdot 0 O E-1 C$ & & THRESH & & 40916 \\
\hline ZR & 91 & $(N, T)$ & $\begin{array}{l}Y \\
Y\end{array}$ & $\begin{array}{l}89 \\
89\end{array}$ & $3.00 E-06$ & & THRESH & & 40917 \\
\hline ZR & 92 & $(N, G)$ & $\begin{array}{l}Z R \\
Z R\end{array}$ & $\begin{array}{l}93 \\
93\end{array}$ & $\begin{array}{l}1.5 \quad E+5 \\
4.00 E-09\end{array}$ & $Y$ & $\begin{array}{l}\text { CoDK } \\
.01960\end{array}$ & $\begin{array}{l}0.00000 \\
T M 102\end{array}$ & 40922 \\
\hline ZR & 92 & $(N, P)$ & $\begin{array}{l}Y \\
Y\end{array}$ & $\begin{array}{l}92 \\
92\end{array}$ & $\begin{array}{l}3.54 \\
1.00 E-08\end{array}$ & $H$ & $\begin{array}{l}\text { THRESH } \\
1.44600\end{array}$ & TM102 & 40923 \\
\hline$Z R$ & 92 & $(N, A)$ & $\begin{array}{l}S R \\
S R\end{array}$ & $\begin{array}{l}89 \\
89\end{array}$ & $\begin{array}{l}50.52 \\
3.00 E-10\end{array}$ & $D$ & $\begin{array}{r}\text { BNL }-325 \\
.58200\end{array}$ & $E N 2 \ddot{23}^{.00008}$ & 40924 \\
\hline
\end{tabular}


TABLE II - CONTINUEO

\begin{tabular}{|c|c|c|c|c|c|c|c|c|c|}
\hline Z R & 92 & $(N, N P+C)$ & $\begin{array}{l}Y \\
y\end{array}$ & $\begin{array}{l}91 \\
91\end{array}$ & $\begin{array}{l}* \\
1.00 E-09\end{array}$ & & THRESH & & 40925 \\
\hline ZR & 92 & $(N, T)$ & $\begin{array}{l}Y \\
Y\end{array}$ & $\begin{array}{l}90 \\
90\end{array}$ & 3. & & THR E SH & & 40927 \\
\hline $2 R$ & 93 & $(N, P)$ & $\begin{array}{l}c \\
Y \\
Y\end{array}$ & 93 & $\begin{array}{l}10.1 \\
5.00 E-07\end{array}$ & $H$ & $\begin{array}{l}\text { THR ESH } \\
1.17300\end{array}$ & T4102 & 40933 \\
\hline ZR & 93 & $(N, A)$ & $\begin{array}{l}S R \\
S R\end{array}$ & $\begin{array}{l}90 \\
90\end{array}$ & $29.00 E-11$ & $Y$ & $\begin{array}{l}\text { THR ESH } \\
.19600\end{array}$ & $\begin{array}{l}0.00000 \\
T M 102\end{array}$ & 40934 \\
\hline$Z R$ & 93 & $(N, N P+D)$ & $\begin{array}{l}\text { Lo } \\
4 \\
4\end{array}$ & $\begin{array}{l}9 ? \\
92 \\
83\end{array}$ & $\begin{array}{l}3.54 \\
1.30 E-09\end{array}$ & $H$ & $\begin{array}{l}\text { THRE SH } \\
1.44600\end{array}$ & TM102 & 40935 \\
\hline$Z R$ & 93 & $(N, V A)$ & $\begin{array}{l}S R \\
S R \\
Y\end{array}$ & $\begin{array}{l}89 \\
89 \\
91\end{array}$ & $\begin{array}{l}50.52 \\
3.00 \mathrm{E}-10 \\
*\end{array}$ & $D$ & $\begin{array}{c}\text { THPES4 } \\
.58200 \\
\text { THRESH }\end{array}$ & EN223 & 40936 \\
\hline $2 \pi$ & & $(N, 1)$ & Y & 91 & 1. $00 E-09$ & & & & 40937 \\
\hline LR & 94 & $(N, 2 N)$ & $\begin{array}{l}Z R \\
Z R \\
Y\end{array}$ & $\begin{array}{l}93 \\
93 \\
94\end{array}$ & $\begin{array}{l}1.5 \quad E+5 \\
4.90 E-09\end{array}$ & $Y$ & $\begin{array}{l}\text { THRESH } \\
.01760\end{array}$ & $\begin{array}{l}0.00000 \\
T 4102\end{array}$ & 40941 \\
\hline$\angle R$ & 94 & $(\mathrm{~N}, \mathrm{P})$ & Y & $\begin{array}{l}74 \\
94\end{array}$ & 3. OCF-CE & $M$ & $\begin{array}{l}\text { THRESH } \\
1.71700\end{array}$ & EN2: 233386 & 40943 \\
\hline ZR & 94 & $(N, A)$ & $\begin{array}{l}S R \\
S R \\
Y\end{array}$ & $\begin{array}{l}91 \\
91 \\
91\end{array}$ & $\begin{array}{l}9.52 \\
9.30 E-09\end{array}$ & $H$ & $\begin{array}{l}\text { THR ESH } \\
.65500\end{array}$ & $\begin{array}{l}1.02435 \\
T M 102\end{array}$ & 40944 \\
\hline Z R & 94 & $(N, N P+D)$ & $\begin{array}{l}c_{0} \\
Y \\
Y\end{array}$ & 93 & $\begin{array}{l}10.1 \\
5.30 E-09\end{array}$ & $H$ & $\begin{array}{l}\text { THRE S4 } \\
1.17300\end{array}$ & $T M 102$ & 40945 \\
\hline$Z R$ & 94 & $(N, N A)$ & $\begin{array}{l}\text { SR } \\
\text { SR }\end{array}$ & 90 & $\begin{array}{l}27 . \\
3.0 O E-11\end{array}$ & $Y$ & $\begin{array}{l}\text { THRESH } \\
.19600\end{array}$ & $\begin{array}{l}0.0 \mathrm{CCOC} \\
T M 102\end{array}$ & 40946 \\
\hline$\angle R$ & 94 & $(N, T)$ & $\begin{array}{l}y \\
y \\
y\end{array}$ & $\begin{array}{l}72 \\
92\end{array}$ & $\begin{array}{l}3.54 \\
1.00 E-08\end{array}$ & 4 & $\begin{array}{l}\text { THR ESH } \\
1.44600\end{array}$ & TM102 & 40947 \\
\hline$Z R$ & 95 & $(N, D)$ & $\begin{array}{l}C^{2} \\
Y\end{array}$ & $\begin{array}{l}95 \\
95\end{array}$ & $\begin{array}{l}10.4 \\
2.00 E-0 t\end{array}$ & $M$ & $\begin{array}{l}\text { THR ESH } \\
1.74500\end{array}$ & E.V $23^{432493}$ & 40953 \\
\hline ZR & 95 & $(N, A)$ & $\begin{array}{l}\text { SR } \\
\text { SR } \\
Y\end{array}$ & $9 ?$ & $\begin{array}{l}2.71 \\
1.00 F-03\end{array}$ & $H$ & $\begin{array}{l}\text { THRESH } \\
.19500\end{array}$ & $\begin{array}{l}1.32514 \\
\mathrm{~T} 102\end{array}$ & 40954 \\
\hline$Z R$ & 95 & $(N, N P+D)$ & $\begin{array}{l}C^{2} \\
Y \\
Y\end{array}$ & $\begin{array}{l}94 \\
94\end{array}$ & 3. JOE -OS & $\mu^{M}$ & $\begin{array}{l}\text { THRE SH } \\
1.71700\end{array}$ & EV 223 & 40955 \\
\hline $2 P$ & 95 & $(N, N \Delta)$ & $\begin{array}{l}S R \\
S R\end{array}$ & 91 & $\begin{array}{l}7.52 \\
9.00 E-\mathrm{CQ}\end{array}$ & $H$ & $\begin{array}{l}\text { THRESH } \\
.55520\end{array}$ & $\begin{array}{l}1.02485 \\
\text { TY } 102\end{array}$ & 40956 \\
\hline ZR & 95 & $(N, T)$ & $\begin{array}{l}Y \\
y\end{array}$ & $\begin{array}{l}93 \\
93\end{array}$ & $\begin{array}{l}10.1 \\
5.00 E-00\end{array}$ & $H$ & $\begin{array}{l}\text { THRESH } \\
1.173 \mathrm{CC}\end{array}$ & TM102 & 40957 \\
\hline
\end{tabular}




\begin{tabular}{|c|c|c|c|c|c|c|c|c|c|c|}
\hline$Z \mathbf{R}$ & 96 & $(N, 2 N)$ & $\begin{array}{l}Z R \\
Z R\end{array}$ & $\begin{array}{l}95 \\
95\end{array}$ & $\begin{array}{l}54.0 \\
1.00 E-09\end{array}$ & D & $\begin{array}{l}\text { THRESH } \\
.11500\end{array}$ & $T 4102$ & 40961 & 0 \\
\hline$Z R$ & 95 & $(N, G)$ & $\begin{array}{l}Z R \\
Z R\end{array}$ & $\begin{array}{l}97 \\
97\end{array}$ & $\begin{array}{l}16.8 \\
3.00 E-09\end{array}$ & $H$ & $\begin{array}{l}030 k \\
.69900\end{array}$ & $T M 102$ & 40962 & 0 \\
\hline$Z R$ & 96 & $(N, D)$ & $\begin{array}{l}y \\
Y\end{array}$ & $\begin{array}{l}76 \\
95\end{array}$ & 3. $O C E-O E$ & 5 & $\begin{array}{l}\text { THP ESH } \\
2.40300\end{array}$ & $\begin{array}{l}0.00000 \\
E \vee 223\end{array}$ & 40963 & 0 \\
\hline $2 P$ & 96 & $(N, A)$ & $\begin{array}{l}S R \\
S R\end{array}$ & $\begin{array}{l}93 \\
93\end{array}$ & $\begin{array}{l}7.5 \\
3.5 O E-O S\end{array}$ & M & $\begin{array}{l}\text { THRESH } \\
1.16100\end{array}$ & $\begin{array}{l}2.12967 \\
\text { EN223 }\end{array}$ & 40964 & 0 \\
\hline ZR & 96 & $(N, N P+D)$ & $\begin{array}{l}Y \\
y\end{array}$ & $\begin{array}{l}95 \\
95\end{array}$ & $\begin{array}{l}10.4 \\
3.00 E-O S\end{array}$ & M & $\begin{array}{l}\text { THRE SH } \\
1.74500\end{array}$ & EN 223 & 40965 & 0 \\
\hline$Z R$ & $7 t$ & $(v, N \Delta)$ & $\begin{array}{l}S P \\
S R\end{array}$ & $\begin{array}{l}92 \\
9 ?\end{array}$ & $\begin{array}{l}2.71 \\
1.00 E-08\end{array}$ & $H$ & $\begin{array}{l}\text { THEEST } \\
.19500\end{array}$ & $\begin{array}{l}1.32514 \\
T 4102\end{array}$ & 40966 & 0 \\
\hline$Z R$ & 95 & $(N, T)$ & $\begin{array}{l}Y \\
Y\end{array}$ & $\begin{array}{l}94 \\
94\end{array}$ & $\begin{array}{l}18 . \\
3.00 E-06\end{array}$ & $M$ & $\begin{array}{l}\text { THR ESH } \\
1.71700\end{array}$ & EN 223 & 40967 & 0 \\
\hline NB & 92 & $(N, 2 N)$ & $\begin{array}{l}N B \\
N B\end{array}$ & $\begin{array}{l}91 \\
91\end{array}$ & $1.00 E-10$ & & THR ESH & & 41921 & 0 \\
\hline Ne & 92 & $(N, A)$ & $\begin{array}{l}Y \\
Y\end{array}$ & $\begin{array}{l}89 \\
89\end{array}$ & $3.00 E-75$ & & THRESH & & 41924 & 0 \\
\hline NB & 92 & $(N, N A)$ & $\begin{array}{l}Y \\
Y\end{array}$ & $\begin{array}{l}88 \\
88\end{array}$ & $1.00 E-10$ & & THPESH & & 41926 & $c$ \\
\hline NB & 92 & $(N, T)$ & $\begin{array}{l}Z R \\
Z R \\
Y\end{array}$ & $\begin{array}{l}90 \\
90 \\
90\end{array}$ & * $3.00 E-05$ & & THRESH & & 41927 & $\mathrm{c}$ \\
\hline NB & 92 & $(N, H E-3)$ & $\begin{array}{l}Y \\
Y\end{array}$ & $\begin{array}{l}90 \\
90\end{array}$ & $3.00 E-07$ & & THRESH & & 41929 & 0 \\
\hline $\mathrm{NB}$ & 93 & $(N, N)$ & $\begin{array}{l}\mathrm{NB} \\
\mathrm{NB}\end{array}$ & $\begin{array}{l}93 M \\
93 M\end{array}$ & $\begin{array}{l}13.6 \\
4.00 E-09\end{array}$ & $Y$ & $\begin{array}{c}\text { FRNC-TH-22 } \\
.03010\end{array}$ & $\begin{array}{l}280.00000 \\
\triangle E R E\end{array}$ & 41930 & 0 \\
\hline NB & 93 & $(N, 2 N)$ & $\begin{array}{l}\text { NB } \\
\text { NB }\end{array}$ & $\begin{array}{l}92 M \\
92 M\end{array}$ & $\begin{array}{l}10.13 \\
3.70 E-C 7\end{array}$ & $\begin{array}{l}D \\
0 K\end{array}$ & $\begin{array}{c}\text { ENDF / } 19-\text { IV } \\
.01260\end{array}$ & DK ${ }^{.92186}$ & 41931 & 0 \\
\hline N B & 93 & $(N, G)$ & $\begin{array}{l}\text { NB } \\
\text { NB }\end{array}$ & $\begin{array}{l}94 \\
94\end{array}$ & $\begin{array}{l}2.0 \quad E+4 \\
2.00 E-00\end{array}$ & $\begin{array}{l}Y \\
\text { OK }\end{array}$ & $\begin{array}{c}\text { ENDF / B - IV } \\
.16700\end{array}$ & $\begin{array}{l}1.57360 \\
\triangle E R E\end{array}$ & 41932 & 0 \\
\hline NB & 93 & $(N, P)$ & $\begin{array}{l}2 R \\
2 R\end{array}$ & $\begin{array}{l}93 \\
93\end{array}$ & $\begin{array}{l}1.5 \quad E+6 \\
4.0 O E-C 9\end{array}$ & $Y$ & $\begin{array}{c}\text { ENDF/B-I V } \\
.01960\end{array}$ & $\begin{array}{l}0.00000 \\
T M 102\end{array}$ & 41933 & 0 \\
\hline NB & 93 & $(N, A)$ & $\begin{array}{l}Y \\
Y\end{array}$ & $\begin{array}{l}90 \\
90\end{array}$ & $\begin{array}{l}64.0 \\
3.00 E-09\end{array}$ & $H$ & $\begin{array}{c}\text { EN DF / B - IV } \\
.93100\end{array}$ & $T 4102$ & 41934 & 0 \\
\hline NB & 93 & $(N, N A)$ & $\begin{array}{l}Y \\
Y\end{array}$ & $\begin{array}{l}89 \\
89\end{array}$ & 3. $3.00 E-06$ & & THRE SH & & 41936 & 0 \\
\hline
\end{tabular}


TAGLE II - CONTINUEO

\begin{tabular}{|c|c|c|c|c|c|c|c|c|c|}
\hline NB & 93 & $(N, H E-3)$ & $\begin{array}{l}Y \\
y\end{array}$ & 91 & *. $1.00 E-09$ & & THRESH & & 41939 \\
\hline NB & 94 & $(N, ? N)$ & $\begin{array}{l}N B \\
\text { NB }\end{array}$ & $\begin{array}{l}93 \\
93\end{array}$ & * $1.00 E-10$ & & THRESH & & 41941 \\
\hline NB & 94 & $(N, A)$ & $\begin{array}{l}Y \\
Y\end{array}$ & $\begin{array}{l}91 \\
91\end{array}$ & * $1.00 F-09$ & & THRESH & & 41944 \\
\hline NB & 94 & $(N, N P+D)$ & $\begin{array}{l}2 R \\
Z R\end{array}$ & $\begin{array}{l}93 \\
93\end{array}$ & $\begin{array}{l}1.5 \quad E+6 \\
4.00 E-09\end{array}$ & Y & $\begin{array}{l}\text { THRESH } \\
.01960\end{array}$ & $\begin{array}{l}0.00000 \\
T M 102\end{array}$ & 41945 \\
\hline NG & 94 & $(N, N \Delta)$ & $\begin{array}{l}Y \\
Y\end{array}$ & $\begin{array}{l}93 \\
90\end{array}$ & * $3.00 E-09$ & & THRESH & & 41946 \\
\hline MO & 100 & $(N, 2 N)$ & $\begin{array}{l}M T \\
M O\end{array}$ & $\begin{array}{l}99 \\
90\end{array}$ & $\begin{array}{l}66.02 \\
7.00 E-C 7\end{array}$ & $H$ & $\begin{array}{l}\text { THRESH } \\
.38500\end{array}$ & $T M 102^{15380}$ & 42001 \\
\hline MO & 100 & $(N, G)$ & $\begin{array}{l}M O \\
M D\end{array}$ & $\begin{array}{l}101 \\
101\end{array}$ & $\begin{array}{l}14.6 \\
3.00 E-06\end{array}$ & M & $\begin{array}{l}\text { COOK } \\
.59500\end{array}$ & EN 223 & 42002 \\
\hline Mo & 100 & $(N, P)$ & $\begin{array}{l}N B \\
N B\end{array}$ & $\begin{array}{l}103 \\
100\end{array}$ & $\begin{array}{l}* \\
3.00 \mathrm{E}-06\end{array}$ & & THRE SH & & 42003 \\
\hline MO & 100 & $(N, \Delta)$ & $\begin{array}{l}Z R \\
Z R\end{array}$ & $\begin{array}{l}97 \\
97\end{array}$ & $\begin{array}{l}16.8 \\
3.50 E-09\end{array}$ & $H$ & $\begin{array}{l}\text { THRESH } \\
.69900\end{array}$ & $T M 1 \dot{02}$ & 42004 \\
\hline MO & 100 & $(N, N P+D)$ & $\begin{array}{l}\text { NB } \\
\text { NB }\end{array}$ & $\begin{array}{l}99 \\
99\end{array}$ & 3.0OE-06 & & THRESH & & 42005 \\
\hline $\mathrm{ma}$ & 100 & $(N, T)$ & $\begin{array}{l}\text { NB } \\
\text { N3 }\end{array}$ & $\begin{array}{l}98 \\
98\end{array}$ & * $3.00 \mathrm{E}-0 \mathrm{~S}$ & & THRE SH & & 42007 \\
\hline MD & 92 & $(N, 2 N)$ & $\begin{array}{l}M Q \\
M Q\end{array}$ & $\begin{array}{l}91 \\
91\end{array}$ & $3.00 E-C E$ & & THRESH & & 42921 \\
\hline MO & 9? & $(N, G)$ & MO & $\begin{array}{l}93 \\
93\end{array}$ & $\begin{array}{l}3.5 \quad E+3 \\
1.00 E-10\end{array}$ & $Y$ & $\begin{array}{l}\text { BEN ZI } \\
0.00000\end{array}$ & $\begin{array}{l}0.00000 \\
\text { TISOT }\end{array}$ & 42922 \\
\hline MO & 92 & $(N, P)$ & $\begin{array}{l}N B \\
N B\end{array}$ & $\begin{array}{l}92 \\
9 ?\end{array}$ & $3.50 E-07$ & $D K$ & THR ESH & & 42923 \\
\hline MO & 92 & $(N, A)$ & $\begin{array}{l}Z R \\
Z R\end{array}$ & $\begin{array}{l}89 \\
89\end{array}$ & $\begin{array}{l}* \\
1.00 E-10\end{array}$ & & THRESH & & 42924 \\
\hline ME: & 92 & $(N, N P+D)$ & $\begin{array}{l}\text { NS } \\
\text { NS }\end{array}$ & $\begin{array}{l}91 \\
91\end{array}$ & $1.00 E-10$ & & THRE SH & & 42925 \\
\hline MD & $9 ?$ & $(N, N A)$ & $\begin{array}{l}2 k \\
2 R\end{array}$ & $\begin{array}{l}88 \\
93\end{array}$ & $\begin{array}{l}33.4 \\
1.00 E-10\end{array}$ & $D$ & $\begin{array}{l}\text { THRESH } \\
0.00000\end{array}$ & $\operatorname{TISO} 7$ & 42726 \\
\hline$M$ & $9 ?$ & $(N \cdot T)$ & $\begin{array}{l}A 3 \\
N B\end{array}$ & $\begin{array}{l}90 \\
9,5\end{array}$ & * $1.00 E-10$ & & THRESH & & 42927 \\
\hline
\end{tabular}




\begin{tabular}{|c|c|c|c|c|c|c|c|c|c|}
\hline MO & 93 & $(N, P)$ & $\begin{array}{l}\text { NB } \\
\text { NR }\end{array}$ & $\begin{array}{l}93 \\
93\end{array}$ & $1.00 \mathrm{E}-10$ & & THRE SH & & 42933 \\
\hline MO & 93 & $(N, A)$ & $\begin{array}{l}Z R \\
Z R\end{array}$ & $\begin{array}{l}90 \\
90\end{array}$ & $\begin{array}{l}\text { * } \\
3.00 E-06\end{array}$ & & THR ESH & & 42934 \\
\hline MD & 93 & $(N, N P+D)$ & $\begin{array}{l}\text { NB } \\
\text { NB }\end{array}$ & $\begin{array}{l}92 \\
92\end{array}$ & * $3.50 E-07$ & DK & THRESH & . & 42935 \\
\hline MD & 93 & $(N, N A)$ & $\begin{array}{l}Z R \\
Z R\end{array}$ & $\begin{array}{l}89 \\
89\end{array}$ & $1.00 E-10$ & & THRFS 4 & & 42936 \\
\hline MO & 93 & $(N, T)$ & $\begin{array}{l}\text { NB } \\
\text { NB }\end{array}$ & $\begin{array}{l}91 \\
91\end{array}$ & $1.00 \mathrm{E}-10$ & & THR E SH & & 42937 \\
\hline MO & 94 & $(N, 2 N)$ & MO & $\begin{array}{l}93 \\
93\end{array}$ & ?. OCE-10 & & THRESH & & 42941 \\
\hline MO & 94 & $(N, P)$ & $\begin{array}{l}\mathrm{NB} \\
\mathrm{NB}\end{array}$ & $\begin{array}{l}94 \\
94\end{array}$ & 2.00E-09 & DK & THRESH & & 42943 \\
\hline MO & 94 & $(N, N P+D)$ & $\begin{array}{l}N B \\
N B\end{array}$ & $\begin{array}{l}93 \\
93\end{array}$ & 1. $.30 E-10$ & & THRE SH & & 42945 \\
\hline MO & 94 & $(N, N A)$ & $\begin{array}{l}2 R \\
7 R\end{array}$ & $\begin{array}{l}90 \\
90\end{array}$ & * $3.00 E-75$ & & THRESH & & 42946 \\
\hline MO & 94 & $(N, T)$ & NB & $9 ?$ & $3.50 E-07$ & DK & THRESH & & 42947 \\
\hline MO & 95 & $(N, P)$ & $\mathrm{NB}$ & $\begin{array}{l}95 \\
95\end{array}$ & $3.00 E-09$ & & THRE SH & & 42953 \\
\hline $\mathrm{MO}$ & 95 & $(N, N P+D)$ & $\begin{array}{l}\text { NB } \\
\text { NB }\end{array}$ & 94 & $? .00 E-09$ & DK & THRESH & & 42955 \\
\hline MO & 95 & $(N, T)$ & $\begin{array}{l}\text { NB } \\
\text { NB }\end{array}$ & $\begin{array}{l}93 \\
93\end{array}$ & $1.00 E-10$ & & THRESH & & 42957 \\
\hline 40 & 95 & $(N, H E-3)$ & $\begin{array}{l}\text { ZR } \\
Z R\end{array}$ & $\begin{array}{l}93 \\
93\end{array}$ & $\begin{array}{l}1.5 \quad E+5 \\
4.00 E-C 9\end{array}$ & $Y$ & $\begin{array}{l}\text { THR E SH } \\
.01940\end{array}$ & $\begin{array}{l}0.00000 \\
\text { TM } 102\end{array}$ & 42759 \\
\hline MO & 96 & $(N, P)$ & $\begin{array}{l}N B \\
N B\end{array}$ & $\begin{array}{l}96 \\
95\end{array}$ & $\begin{array}{l}23.4 \\
1.5 O E-10\end{array}$ & $\mathrm{H}$ & $\begin{array}{l}\text { THRESH } \\
.24900\end{array}$ & $\begin{array}{l}2.58183 \\
\text { EN } 223\end{array}$ & 42963 \\
\hline$M 0$ & 96 & $(N, A)$ & $\begin{array}{l}Z R \\
Z R\end{array}$ & $\begin{array}{l}93 \\
93\end{array}$ & $\begin{array}{l}1.5 \quad E+6 \\
4.00 E-09\end{array}$ & $Y$ & $\begin{array}{l}\text { THRESH } \\
.01760\end{array}$ & $\begin{array}{l}0.00000 \\
T M 102\end{array}$ & 42954 \\
\hline MO & 96 & $(N, N P+D)$ & $\begin{array}{l}\text { NB } \\
\text { NB }\end{array}$ & $\begin{array}{l}95 \\
95\end{array}$ & 2. OCF-CQ & & THRESH & & 42965 \\
\hline$M D$ & 96 & $(N, T)$ & $\begin{array}{l}N B \\
\because B\end{array}$ & $\begin{array}{l}94 \\
94\end{array}$ & $2.00 E-09$ & DK & THRESH & & 42967 \\
\hline
\end{tabular}




\begin{tabular}{|c|c|c|c|c|c|c|c|c|c|c|c|c|c|c|c|c|}
\hline$c$ & 0 & 0 & 0 & 0 & $\boldsymbol{0}$ & $n$ & 0 & 0 & 0 & 0 & 0 & 0 & 0 & 0 & 0 & 0 \\
\hline$\stackrel{m}{\sim}$ & \begin{tabular}{l}
$n$ \\
$\sim$ \\
$\mathfrak{N}$ \\
\multirow{v}{N}{}
\end{tabular} & $\begin{array}{l}0 \\
\sim \\
N \\
N\end{array}$ & 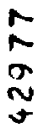 & 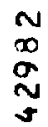 & 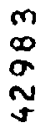 & $\begin{array}{l}\mathbf{w} \\
\infty \\
\infty \\
\sim\end{array}$ & \begin{tabular}{l}
$n$ \\
$\infty$ \\
$\alpha$ \\
$\sim$ \\
\multirow{n}{*}{}
\end{tabular} & \begin{tabular}{l}
$\sim$ \\
$\infty$ \\
$\sigma$ \\
\multirow{\sigma}{*}{}
\end{tabular} & $\begin{array}{l}m \\
\alpha \\
a \\
n \\
j\end{array}$ & $\begin{array}{l}\text { n } \\
\sigma \\
\sigma \\
\mathbb{v} \\
v\end{array}$ & 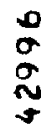 & 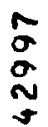 & $\begin{array}{l}n \\
\alpha \\
m \\
q\end{array}$ & 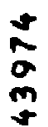 & $\begin{array}{l}p \\
\sigma \\
m\end{array}$ & 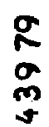 \\
\hline
\end{tabular}

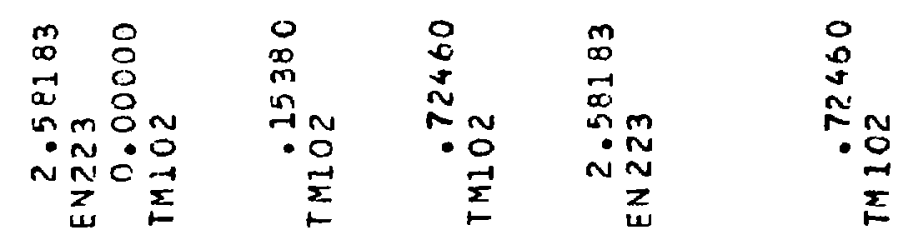

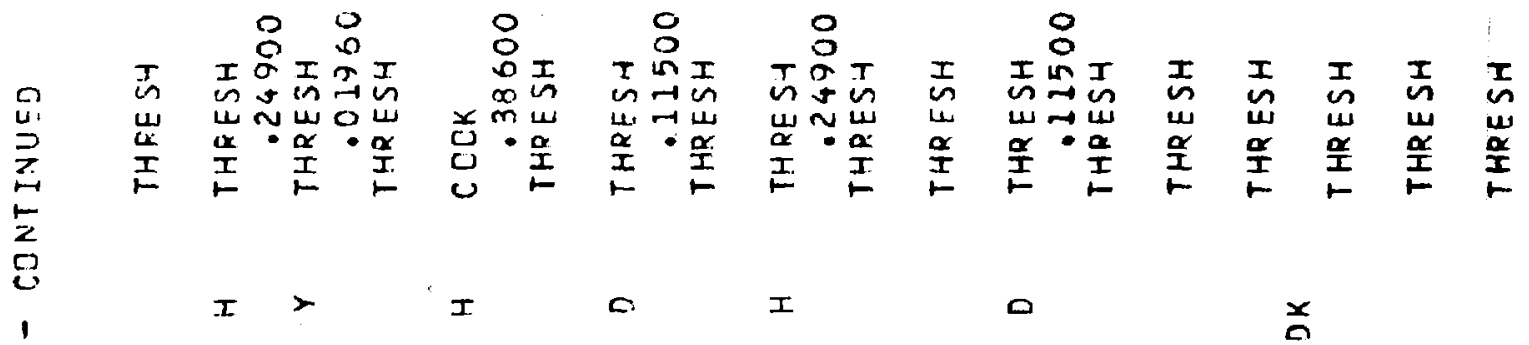

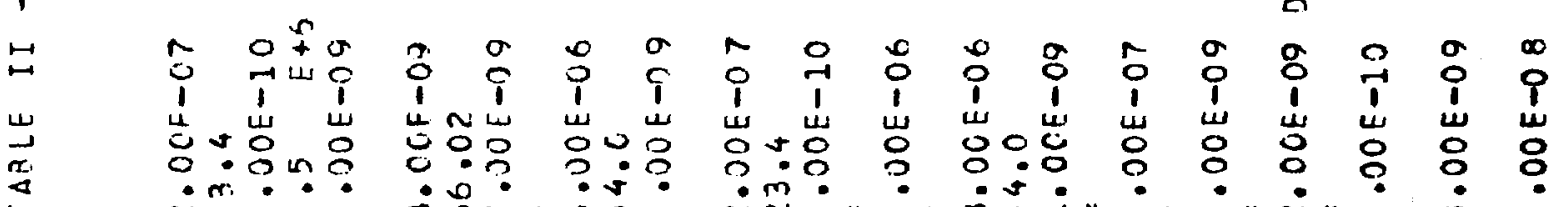

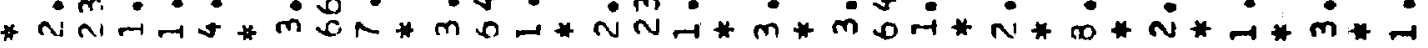

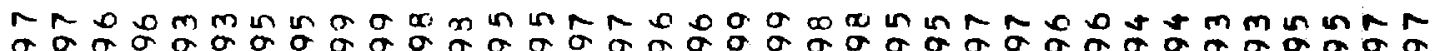

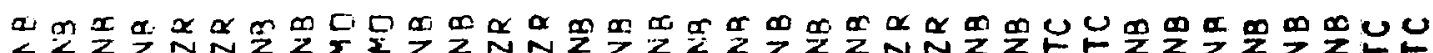

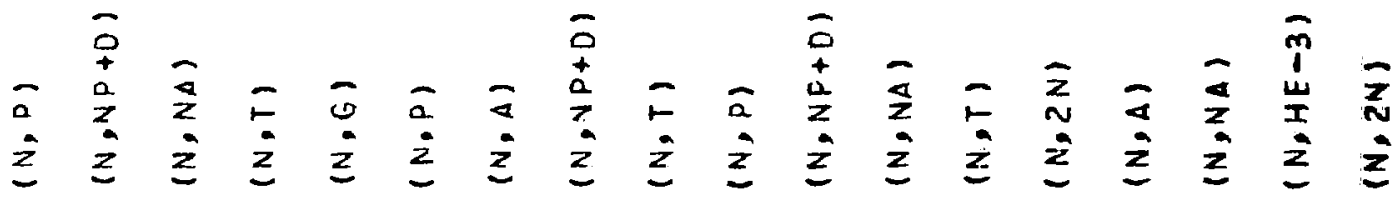

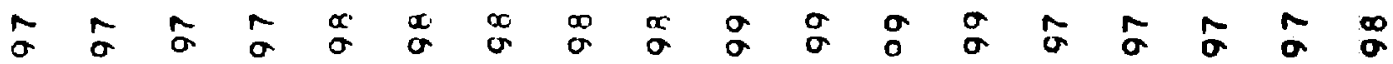

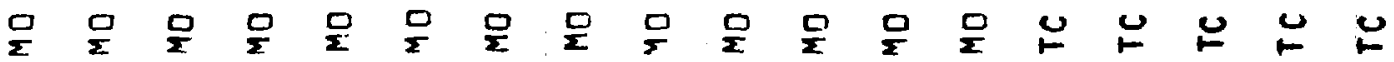




\begin{tabular}{|c|c|c|c|c|c|c|c|c|c|c|c|c|c|c|c|c|c|}
\hline 0 & $\sigma$ & 0 & 0 & 0 & 0 & 0 & $\boldsymbol{O}$ & 0 & 0 & 0 & 0 & 0 & 0 & $\boldsymbol{0}$ & 0 & 0 & 0 \\
\hline $\begin{array}{l}+\vec{w} \\
\dot{0} \\
\sigma \\
m\end{array}$ & $\begin{array}{l}\infty \\
\infty \\
m\end{array}$ & $\begin{array}{l}\vec{m} \\
\sigma \\
m\end{array}$ & $\begin{array}{l}w \\
0 \\
m \\
m\end{array}$ & $\begin{array}{l}n \\
0 \\
0 \\
m\end{array}$ & $\begin{array}{l}5 \\
\sigma \\
\sigma \\
01 \\
1\end{array}$ & $\begin{array}{l}\omega \\
\sigma \\
\sigma \\
m\end{array}$ & $\begin{array}{l}0 \\
5 \\
5 \\
5\end{array}$ & $\underset{R}{R}$ & $\stackrel{N}{N}$ & $\stackrel{m}{R}$ & $\stackrel{5}{0}$ & $\begin{array}{l}0 \\
0 \\
0\end{array}$ & $\begin{array}{l}N \\
\sigma \\
0\end{array}$ & $\begin{array}{l}m \\
0 \\
\Omega\end{array}$ & $\begin{array}{l}0 \\
0\end{array}$ & $\underset{0}{N}$ & N \\
\hline
\end{tabular}

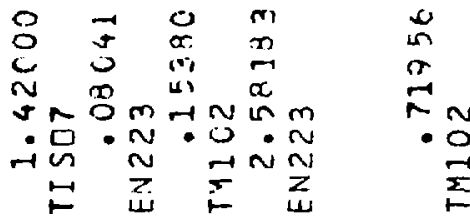

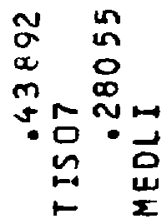

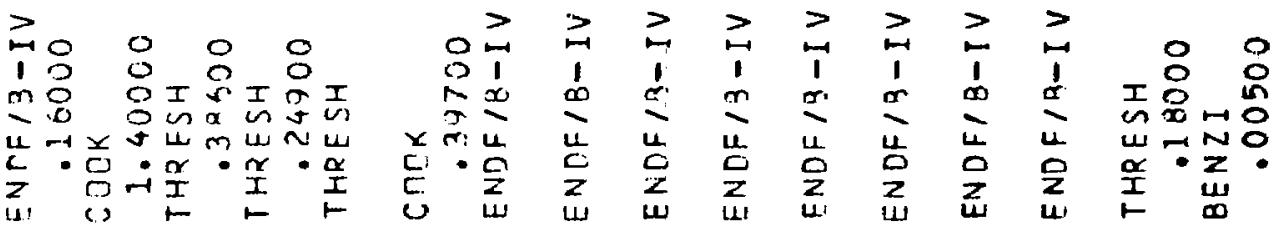

告

$r>m I I$

2.0

$\rightarrow$

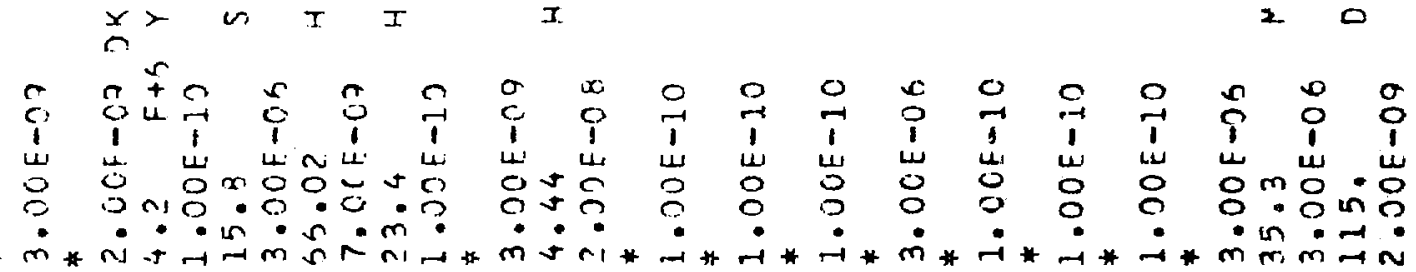

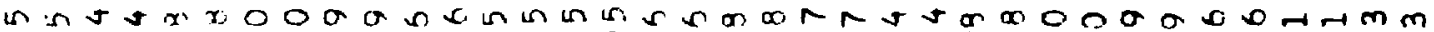

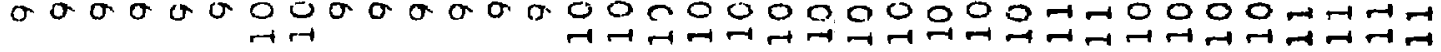

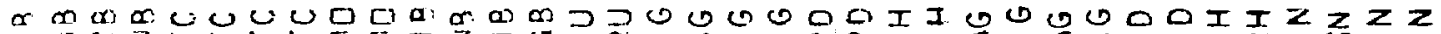

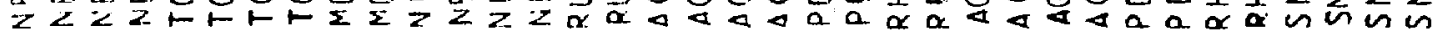

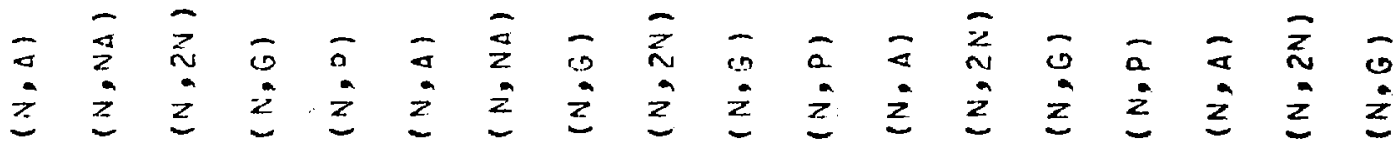

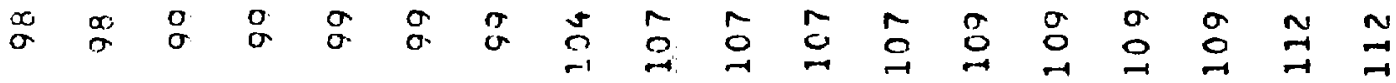

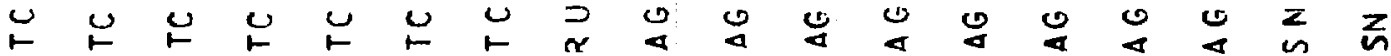




\begin{tabular}{|c|c|c|c|c|c|c|c|c|c|}
\hline$S N$ & 112 & $(N, P)$ & $\begin{array}{l}\text { IN } \\
\text { IN }\end{array}$ & $\begin{array}{l}112 \\
112\end{array}$ & $\begin{array}{l}* \\
3.00 E-06\end{array}$ & & THRESH & & 50123 \\
\hline SN & 112 & $(N, A)$ & $\begin{array}{l}C D \\
C D\end{array}$ & $\begin{array}{l}109 \\
109\end{array}$ & $\begin{array}{l}453 . \\
2.00 E-09\end{array}$ & D & $\begin{array}{l}\text { THRE SH } \\
\quad .02460\end{array}$ & EN 223 & 50124 \\
\hline SN & 112 & $(N, N F+D)$ & $\begin{array}{l}\text { IN } \\
\text { IN }\end{array}$ & $\begin{array}{l}111 \\
111\end{array}$ & 1. $O O F-10$ & & THRESH & & 50125 \\
\hline SN & 112 & $(N, T)$ & $\begin{array}{l}\text { IN } \\
\text { IN }\end{array}$ & $\begin{array}{l}110 \\
110\end{array}$ & 1. & & THPESH & & 50127 \\
\hline SN & 114 & $(N, 2 N)$ & SN & $\begin{array}{l}113 \\
113\end{array}$ & $\begin{array}{l}* \\
2.00 E-09\end{array}$ & & THRESH & & 50141 \\
\hline SN & 114 & $(N, D)$ & IN & $\begin{array}{l}114 \\
114\end{array}$ & 1. $.0 E-13$ & & THRESH & & 50143 \\
\hline$S N$ & 114 & $(N, A)$ & $\begin{array}{l}C D \\
C D\end{array}$ & $\begin{array}{l}111 \\
111\end{array}$ & 3. $30 E-05$ & & THR ESH & & 50144 \\
\hline SN & 114 & $(N, N P+D)$ & $\begin{array}{l}\text { IN } \\
\text { IN }\end{array}$ & $\begin{array}{l}113 \\
113\end{array}$ & $3.00 E-06$ & & THRESH & & 50145 \\
\hline SN & 114 & $(N, T)$ & $\begin{array}{l}\text { IN } \\
\text { IN }\end{array}$ & $\begin{array}{l}112 \\
11 ?\end{array}$ & $3.00 \mathrm{E}-0 \mathrm{~S}$ & & THRESH & & 50147 \\
\hline$S N$ & 115 & $(N, 0)$ & $\begin{array}{l}\text { IN } \\
\text { IN }\end{array}$ & $\begin{array}{l}115 \\
115\end{array}$ & $1.00 E-09$ & & THRESH & & 50153 \\
\hline SN & 115 & $(N, N P+D)$ & $\begin{array}{l}\text { IN } \\
\text { IN }\end{array}$ & $\begin{array}{l}114 \\
114\end{array}$ & 1. $1.00 E-10$ & & THPE SH & & 50155 \\
\hline$S N$ & 115 & $(N, N A)$ & $\begin{array}{l}C D \\
C B\end{array}$ & $\begin{array}{l}111 \\
111\end{array}$ & $3.20 \mathrm{E}-0 \mathrm{~h}$ & & THRFSH & & 50156 \\
\hline$S N$ & 115 & $(N, T)$ & $\begin{array}{l}\text { IN } \\
\text { IN }\end{array}$ & $\begin{array}{l}113 \\
113\end{array}$ & $3.00 E-06$ & & THRESH & & $5 C 157$ \\
\hline SN & 116 & $(N, G)$ & $\begin{array}{l}\text { SN } \\
\text { SN }\end{array}$ & $\begin{array}{l}117 \\
117\end{array}$ & 1.00E-10 & & CJOK & & 50162 \\
\hline SN & 116 & $(N, P)$ & $\begin{array}{l}\text { IN } \\
\text { IN }\end{array}$ & $\begin{array}{l}115 \\
11 t\end{array}$ & $3.30 E-\cap 6$ & & THRSSH & & 50163 \\
\hline SN & 116 & $(N, \Delta)$ & $\begin{array}{l}C D \\
C D\end{array}$ & $\begin{array}{l}112 \\
113\end{array}$ & 1. $00 E-10$ & & THRESH & & 50164 \\
\hline SN & 116 & $(N, N P+D)$ & IN & $\begin{array}{l}115 \\
115\end{array}$ & I. JOE-09 & & THRESH & & 50165 \\
\hline SN & 116 & $(N, T)$ & $\begin{array}{l}\text { IN } \\
\text { IN }\end{array}$ & $\begin{array}{l}114 \\
114\end{array}$ & * $1.0 C E-10$ & & THRESH & & 50167 \\
\hline
\end{tabular}


TABLE II - CONTINUED

\begin{tabular}{|c|c|c|c|c|c|c|c|}
\hline$S N$ & 117 & $(N, P)$ & $\begin{array}{l}\text { IN } \\
\text { IN }\end{array}$ & $\begin{array}{l}117 \\
117\end{array}$ & $\begin{array}{l}* \\
3.00 E-0 h\end{array}$ & & THRESH \\
\hline SN & 117 & $(N, N P+D)$ & IN & 116 & & & $T H R \subseteq S H$ \\
\hline$S N$ & 117 & $(N, N A)$ & $\begin{array}{l}1 \\
C D \\
C D\end{array}$ & $\begin{array}{l}1 \\
113 \\
113\end{array}$ & $1.00 E-12$ & & THRESH \\
\hline$S N$ & 117 & $(N, T)$ & $\begin{array}{l}\text { IN } \\
\text { IN }\end{array}$ & $\begin{array}{l}115 \\
115\end{array}$ & $\begin{array}{l}* \\
1.00 E-09\end{array}$ & & THRE SH \\
\hline SN & 118 & $(N, 2 N)$ & $\begin{array}{l}\text { SN } \\
\text { SN }\end{array}$ & $\begin{array}{l}117 \\
117\end{array}$ & $1700 \mathrm{E}-10$ & & THRESH \\
\hline$S N$ & 118 & $(N, G)$ & SN & $\begin{array}{l}119 \\
119\end{array}$ & $1.00 E-10$ & & COOK \\
\hline SN & 118 & $(N, P)$ & $\begin{array}{l}\text { IN } \\
\text { IN }\end{array}$ & $\begin{array}{l}119 \\
119\end{array}$ & $3.00 E-06$ & 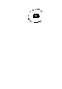 & THRE SH \\
\hline$S N$ & 119 & $(N, \Delta)$ & $\begin{array}{l}C D \\
C D\end{array}$ & $\begin{array}{l}115 \\
115\end{array}$ & 6. & . & THRFSH \\
\hline$S N$ & 118 & $(N, N P+D)$ & IN & $\begin{array}{l}117 \\
117\end{array}$ & $3.00 E-06$ & & THRESH \\
\hline SN & 113 & $(N, T)$ & IN & 116 & $3.00 E-05$ & & THPE SH \\
\hline SN & 117 & $(N, P)$ & IN & $\begin{array}{l}119 \\
119\end{array}$ & $3.2 C E-O G$ & & THRES - \\
\hline $\mathrm{SN}$ & 19 & $(N, N P+D)$ & IN & 113 & $3.00 E-05$ & $\therefore$ & THRESH \\
\hline SN & 119 & $(N, N A)$ & $\begin{array}{l}C D \\
C D\end{array}$ & $\begin{array}{l}115 \\
115\end{array}$ & 6. COE-CP & & THRESH \\
\hline$S N=$ & 129 & $(N, T)$ & IN & $\begin{array}{l}117 \\
117\end{array}$ & * $3.0 O E-06$ & & THRESH = \\
\hline $\mathrm{SN}$ & $12 \%$ & $(N, ? N)$ & $\begin{array}{l}S N \\
S N\end{array}$ & $\begin{array}{l}119 \\
117\end{array}$ & * $1.00 E-10$ & & THRE SH \\
\hline SN & 120 & $(N, G)$ & $\begin{array}{l}S N \\
S N\end{array}$ & $\begin{array}{l}121 \\
1 \geq 1\end{array}$ & 1. UCF-1n & & SOCK \\
\hline SN & 120 & $(N, P)$ & IN & $\begin{array}{l}120 \\
1 \geq 0\end{array}$ & $3.00 E-05$ & & THRESH \\
\hline SN & 120 & $\begin{array}{c}(N, A) \\
=\end{array}$ & $\begin{array}{l}C D \\
C D\end{array}$ & $\begin{array}{l}117 \\
117\end{array}$ & $1.00 E-10$ & in & THRESH \\
\hline
\end{tabular}

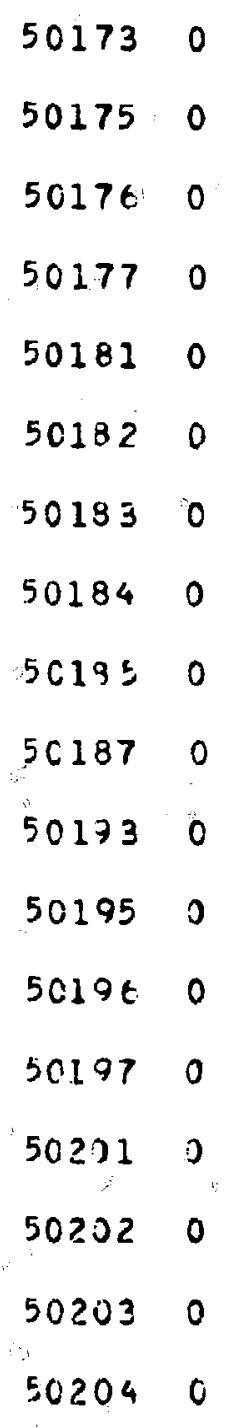




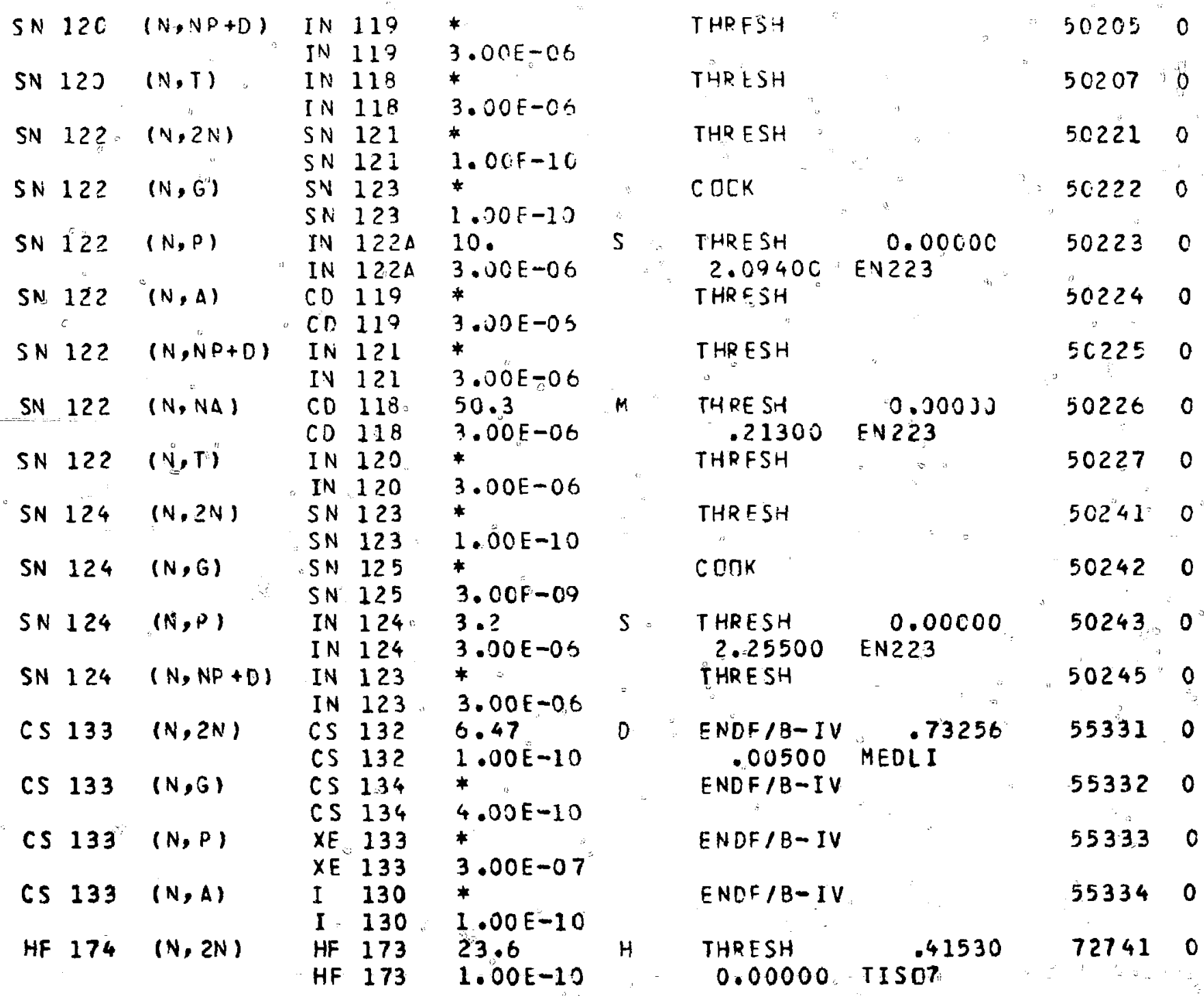




\begin{tabular}{|c|c|c|c|c|c|c|c|c|c|}
\hline HF & 174 & $(N, B)$ & $\begin{array}{l}\mathrm{HF} \\
\mathrm{HF}\end{array}$ & $\begin{array}{l}175 \\
175\end{array}$ & $\begin{array}{l}70 . \\
1.00 F-10\end{array}$ & 0 & $\begin{array}{r}\text { ENDF } 19-1 \\
.09530\end{array}$ & $A E R \dot{E}^{34422}$ & $7274 ?$ \\
\hline HF & 174 & $(N, P)$ & $\begin{array}{l}\text { LU } \\
\text { LU }\end{array}$ & $\begin{array}{l}174 \\
174\end{array}$ & $\begin{array}{l}3.3 \\
1.5 O E-10\end{array}$ & Y & $\begin{array}{l}\text { THRESH } \\
0.00000\end{array}$ & TISO7 & 72743 \\
\hline HF & 176 & $(N, 2 N)$ & $\begin{array}{l}H F \\
H F\end{array}$ & $\begin{array}{l}175 \\
175\end{array}$ & $\begin{array}{l}70 . \\
1.00 E-10\end{array}$ & D & $\begin{array}{r}\text { ENDF / } 3-1 \\
.09630\end{array}$ & $A E R \dot{E}^{.344 ? 2}$ & 72761 \\
\hline HF & 177 & $(N, P)$ & LU & $\begin{array}{l}177 M \\
177 M\end{array}$ & $\begin{array}{l}161 . \\
1.0 O E-10\end{array}$ & D & $\begin{array}{l}\text { THRESH } \\
.051 D O\end{array}$ & TIS:07 & 72773 \\
\hline HF & 180 & $(N, G)$ & $\begin{array}{l}H F \\
H F\end{array}$ & $\begin{array}{l}131 \\
181\end{array}$ & $\begin{array}{l}42.4 \\
1.00 E-07\end{array}$ & $\Gamma$ & $\begin{array}{r}\text { ENDF/B-1 } \\
.21800\end{array}$ & $\triangle E R \dot{E}^{.52421}$ & 72802 \\
\hline TA & 181 & $(N, 2 N)$ & $\begin{array}{l}\text { TA } \\
\text { TA }\end{array}$ & $\begin{array}{l}180 \mathrm{M} \\
180 \mathrm{M}\end{array}$ & $\begin{array}{l}3.12 \\
1.0 C F-10\end{array}$ & 4 & $\begin{array}{c}\text { ENDF } 13-1 \mathrm{~V} \\
.03500\end{array}$ & TISO? & 73911 \\
\hline TA & 181 & $(N, G)$ & $\begin{array}{l}\text { TA } \\
\text { TA }\end{array}$ & $\begin{array}{l}182 \\
182\end{array}$ & $\begin{array}{l}115 . \\
7.00 E-10\end{array}$ & {[} & $\begin{array}{c}\text { ENDF/3-JV } \\
.22400\end{array}$ & $\begin{array}{l}1.36620 \\
\triangle E R E\end{array}$ & 73812 \\
\hline TA & 181 & $(N, P)$ & $\begin{array}{l}\mathrm{HF} \\
\mathrm{HF}\end{array}$ & $\begin{array}{l}281 \\
181\end{array}$ & 42.4 & 0 & $\begin{array}{c}\text { ENDF/B-IV } \\
.2180 \mathrm{C}\end{array}$ & $\triangle E R \dot{E}^{.52421}$ & 73813 \\
\hline TA & 181 & $(N, A)$ & LU & $\begin{array}{l}178 \\
178\end{array}$ & $\begin{array}{l}28.4 \\
3.00 E-76\end{array}$ & $M$ & $\begin{array}{r}\text { ENDF } / 8-3 \\
.73300\end{array}$ & $\operatorname{Tisin} 7^{25449}$ & 73814 \\
\hline TA & 181 & $(N, N P+D)$ & $\begin{array}{l}\text { HF } \\
H F\end{array}$ & $\begin{array}{l}180 \\
180\end{array}$ & 1. OCE-10 & & THRFSH & & 73815 \\
\hline TA & 181 & $(N, N A)$ & $\begin{array}{l}\text { LU } \\
\text { LU }\end{array}$ & $\begin{array}{l}177 \\
177\end{array}$ & $2.00 E-0.9$ & & THRESH & & 73816 \\
\hline TA & 181 & $(N, T)$ & $\begin{array}{l}H F \\
H F\end{array}$ & $\begin{array}{l}179 \\
179\end{array}$ & $1.00 E-1 C$ & & THRESH & & 73817 \\
\hline TA & 182 & $(N, G)$ & $\begin{array}{l}\text { TA } \\
\text { TA }\end{array}$ & $\begin{array}{l}183 \\
183\end{array}$ & $\begin{array}{l}5.0 \\
1.00 E-10\end{array}$ & 0 & $\begin{array}{c}\text { ENDF/B-IV } \\
23000\end{array}$ & TISO7 & 73822 \\
\hline IA & 182 & $(N, A)$ & LU & $\begin{array}{l}179 \\
179\end{array}$ & $4.600-10$ & H & $\begin{array}{c}\text { ENDF } 19-\text { IV } \\
.53000\end{array}$ & $\begin{array}{l}0.00000 \\
\text { TISO7 }\end{array}$ & 13824 \\
\hline $\mathbf{w}$ & 182 & $(N, ? N)$ & $W$ & $\begin{array}{l}181 \\
181\end{array}$ & $\begin{array}{l}121.0 \\
4.00 E-09\end{array}$ & D & $\begin{array}{c}\text { ENDF/B-IV } \\
05180 \\
\text { FNDF/B-TV }\end{array}$ & $\triangle E R \dot{E}^{04612}$ & $190 \leq 1$ \\
\hline $\mathbf{W}$ & 182 & $(N, P)$ & TA & $\begin{array}{l}182 \\
182\end{array}$ & $\begin{array}{l}115 . \\
7.00 E-10\end{array}$ & $D$ & $\begin{array}{c}\text { ENDF/B-IV } \\
22400\end{array}$ & AERE & 74823 \\
\hline$W$ & 186 & $(N, A)$ & $\begin{array}{l}H F \\
H F\end{array}$ & $\begin{array}{l}179 \\
179\end{array}$ & $1.00 E-10$ & & ENDF/B-IV & & 14824 \\
\hline$w$ & 182 & $(N, N A)$ & $\begin{array}{l}\mathrm{HF} \\
\mathrm{HF}\end{array}$ & $\begin{array}{l}178 \\
178\end{array}$ & $1.00 E-10$ & & THRESH & & 74826 \\
\hline
\end{tabular}




\begin{tabular}{|c|c|c|c|c|c|c|c|c|c|}
\hline $\mathbf{W}$ & 182 & $(N, T)$ & $\begin{array}{l}\text { TA } \\
\text { TA }\end{array}$ & $\begin{array}{l}180 \\
180\end{array}$ & * $1.00 E-10$ & & THPE SH & & 74827 \\
\hline$W$ & 183 & $(N, P)$ & $\begin{array}{l}\text { TA } \\
\text { TA }\end{array}$ & $\begin{array}{l}183 \\
183\end{array}$ & $\begin{array}{l}5.0 \\
1.0 O E-10\end{array}$ & D & $\begin{array}{c}\text { ENDF / } / \text { - IV } \\
.23000\end{array}$ & TISO07 & 74833 \\
\hline W & 183 & $(N, A)$ & $\begin{array}{l}H F \\
H F\end{array}$ & $\begin{array}{l}190 \\
180\end{array}$ & $1.00 \mathrm{E}-10$ & & ENDF/3-IV & & 74834 \\
\hline$W$ & 183 & $(N, N P+D)$ & $\begin{array}{l}\text { TA } \\
\text { TA }\end{array}$ & $\begin{array}{l}182 \\
182\end{array}$ & 7. OOE -10 & & ENDF $13-$ I V & & 74835 \\
\hline W & 183 & $(N, N \Delta)$ & $\begin{array}{l}\text { HF } \\
\text { HF }\end{array}$ & $\begin{array}{l}179 \\
179\end{array}$ & 1. $00 E-10$ & & THRESH & & 74836 \\
\hline$W$ & 183 & $(N, 3 N)$ & $h$ & $\begin{array}{l}181 \\
131\end{array}$ & $\begin{array}{l}121.0 \\
4.00 E-00\end{array}$ & D & $\begin{array}{c}\text { ENDF/B-IV } \\
.05130\end{array}$ & $\triangle E Q \dot{E}^{.04 t 12}$ & 74838 \\
\hline$W$ & 184 & $(N, G)$ & $w$ & $\begin{array}{l}185 \\
195\end{array}$ & $\begin{array}{l}75.1 \\
4.0 C F-09\end{array}$ & 0 & $\begin{array}{c}\text { ENDF } 13-I V \\
.12700\end{array}$ & $\triangle E R E$ & 74842 \\
\hline $\mathbf{W}$ & 184 & $(N, P)$ & $\begin{array}{l}T A \\
\text { TA }\end{array}$ & $\begin{array}{l}184 \\
184\end{array}$ & $\begin{array}{l}8.7 \\
1.20 E-10\end{array}$ & $\mathrm{H}$ & $\begin{array}{c}\text { ENDF/B-IV } \\
48000\end{array}$ & $\begin{array}{l}1.67131 \\
T 1507\end{array}$ & 74843 \\
\hline$W$ & 184 & $(N, A)$ & $\begin{array}{l}H F \\
H F\end{array}$ & $\begin{array}{l}181 \\
191\end{array}$ & $\begin{array}{l}42.4 \\
1.00 E-00\end{array}$ & 0 & $\begin{array}{c}\text { ENDF } / B \text { - IV } \\
.21900\end{array}$ & $\triangle E R \dot{E}^{.52421}$ & 74844 \\
\hline$W$ & 184 & $(N, N P+D)$ & $\begin{array}{l}\text { TA } \\
\text { TA }\end{array}$ & $\begin{array}{l}183 \\
183\end{array}$ & $\begin{array}{l}5 . C \\
1.00 E-10\end{array}$ & 2 & $\begin{array}{c}\text { ENDF / B-IV } \\
-23.300\end{array}$ & $\begin{array}{l}.29277 \\
\text { TIS } 07\end{array}$ & 7494 \\
\hline W & 184 & $(N, N A)$ & $\begin{array}{l}H F \\
H F\end{array}$ & $\begin{array}{l}180 \\
180\end{array}$ & $1.00 \mathrm{E}-10$ & & THRESH & & 74846 \\
\hline$W$ & 184 & $(N, T)$ & $\begin{array}{l}\text { TA } \\
\text { TA }\end{array}$ & $\begin{array}{l}182 \\
182\end{array}$ & 7. OOF -10 & & THR ESH & & 74847 \\
\hline$W$ & 186 & $(N, ? N)$ & $w$ & $\begin{array}{l}185 \\
185\end{array}$ & $\begin{array}{l}75.1 \\
4 . J 0 E-J 9\end{array}$ & c & $\begin{array}{c}\text { ENDF } / 3-\text { IV } \\
.12700\end{array}$ & $\triangle E R \dot{E}^{.00002}$ & 74861 \\
\hline 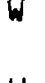 & 186 & $(N, G)$ & $k$ & $\begin{array}{l}137 \\
187\end{array}$ & $\begin{array}{l}23.9 \\
1.30 E-08\end{array}$ & H & $\begin{array}{c}\text { FNOF/R-IV } \\
.23900\end{array}$ & $\triangle E R \dot{E}^{.51 t 11}$ & 74362 \\
\hline$W$ & 186 & $(N, P)$ & TA & $\begin{array}{l}186 \\
186\end{array}$ & $\begin{array}{l}10.5 \\
3.00 F-C S\end{array}$ & $M$ & $\begin{array}{c}\text { ENDF / } 3-\text { IV } \\
.88000\end{array}$ & $\begin{array}{l}1.65: 16 \\
\text { TISO7 }\end{array}$ & 74863 \\
\hline W & 186 & $(N, A)$ & $\begin{array}{l}\text { HF } \\
H F\end{array}$ & $\begin{array}{l}183 \\
183\end{array}$ & $\begin{array}{l}54 . \\
3.00 F-05\end{array}$ & 4 & $\begin{array}{c}\text { ENDF/ } / 9-I V \\
.51000\end{array}$ & TISC7 & 74364 \\
\hline $\mathbf{W}$ & 186 & $(N \cdot N P+D)$ & TA & $\begin{array}{l}185 \\
185\end{array}$ & $\begin{array}{l}49 . \\
3 . J C E-D S\end{array}$ & $M$ & $\begin{array}{c}\text { ENDF/R-IV } \\
\text {. }\end{array}$ & $\begin{array}{l}0.00000 \\
\text { TISD7 }\end{array}$ & 74865 \\
\hline $\mathbf{W}$ & 186 & $(N, N \Delta)$ & $\begin{array}{l}\text { HF } \\
\text { HF }\end{array}$ & $\begin{array}{l}18 ? \\
18 ?\end{array}$ & $\begin{array}{l}7.5+6 \\
1.0 O E-1 C\end{array}$ & $Y$ & $\begin{array}{l}\text { THRESH } \\
.20000\end{array}$ & $\operatorname{TISD7}$ & 74860 \\
\hline
\end{tabular}




\begin{tabular}{|c|c|c|c|c|c|c|c|c|c|}
\hline$W$ & 196 & $(\mathrm{~N}, \mathrm{~T})$ & $\begin{array}{l}\text { TA } \\
\text { TA }\end{array}$ & $\begin{array}{l}184 \\
184\end{array}$ & $\begin{array}{l}3.7 \\
1.00 E-10\end{array}$ & H & $\begin{array}{l}\text { THRES4 } \\
.48000\end{array}$ & $\begin{array}{l}1.67131 \\
\text { TIS07 }\end{array}$ & 74867 \\
\hline RE & 185 & $(N, 2 N)$ & $\begin{array}{l}\text { R.E } \\
\text { RE }\end{array}$ & $\begin{array}{l}184 \\
184\end{array}$ & $1.00 E-10$ & & ENDF/B-IV & & 75651 \\
\hline RE & 185 & $(N, G)$ & $\begin{array}{l}\text { RE } \\
\text { RE }\end{array}$ & $\begin{array}{l}185 \\
186\end{array}$ & $8.00 E-09$ & & ENDF / & & 75832 \\
\hline$R E$ & 185 & $(N, 3 N)$ & $\begin{array}{l}R E \\
Q E\end{array}$ & $\begin{array}{l}193 \\
183\end{array}$ & $\begin{array}{l}70 . \\
5.00 E-09\end{array}$ & D & $\begin{array}{c}\text { ENCF/B-IV } \\
0.00000\end{array}$ & TI SÜ $7781 \mathrm{H}$ & 75858 \\
\hline $\mathrm{E}$ & 187 & $(N, 2 N)$ & $\begin{array}{l}\text { RF } \\
\text { RE }\end{array}$ & $\begin{array}{l}180 \\
135\end{array}$ & $3.00 E-c 9$ & & ENCF/B-IV & & 75871 \\
\hline RE & 187 & $(N, G)$ & $\begin{array}{l}\text { RE } \\
R E\end{array}$ & $\begin{array}{l}188 \\
189\end{array}$ & $6.00 E-03$ & & FNDF/E-IV & & 75872 \\
\hline$A U$ & 197 & $(N, 2 N)$ & $\Delta U$ & $\begin{array}{l}196 \\
196\end{array}$ & 2. OOE-C3 & & ENDF/I-IV & & 79971 \\
\hline$\Delta U$ & 197 & $(N, G)$ & $A U$ & $\begin{array}{l}199 \\
198\end{array}$ & * $8.00 F-09$ & & EVPF/3-IV & & 79972 \\
\hline$\Delta U$ & 197 & $(N, P)$ & $\begin{array}{l}\text { PT } \\
\text { DT }\end{array}$ & $\begin{array}{l}197 \\
197\end{array}$ & 2. & & ENDF/B-IV & & 79973 \\
\hline PB & 204 & $(N, 2 N)$ & $\begin{array}{l}\mathrm{PP} \\
\mathrm{PB}\end{array}$ & $\begin{array}{l}203 \\
203\end{array}$ & * $3.00 E-08$ & & THRESH & & 82041 \\
\hline PB & 204 & $(N, P)$ & $\begin{array}{l}T L \\
T L\end{array}$ & $\begin{array}{l}204 \\
204\end{array}$ & $\begin{array}{l}3.77 \\
9.00 E-10\end{array}$ & $\mathbf{Y}$ & $\begin{array}{l}\text { THRESH } \\
.30000\end{array}$ & T I507 & 92043 \\
\hline P B & 204 & $(N, T)$ & $\begin{array}{l}T L \\
T L \\
P B\end{array}$ & $\begin{array}{l}202 \\
202 \\
205\end{array}$ & $\begin{array}{l}12.23 \\
8.00 E-09 \\
1.4 \quad E+7\end{array}$ & $\mathrm{H}$ & $\begin{array}{l}\text { THRE SH } \\
\text { O.00000 } \\
\text { THP }\end{array}$ & TI SO & 82047 \\
\hline PB & 206 & $(N, P)$ & $\begin{array}{l}\text { PB } \\
T L \\
T L\end{array}$ & $\begin{array}{l}205 \\
205 \\
206 \\
206\end{array}$ & $\begin{array}{l}1.4 \\
1.00 E-10 \\
3.00 E-06\end{array}$ & $\mathbf{r}$ & $\begin{array}{l}\text { O.0ODOO } \\
\text { THRESH }\end{array}$ & TISO7 & 82063 \\
\hline PB & 206 & $(N, A)$ & $\begin{array}{l}H G \\
H G\end{array}$ & $\begin{array}{l}203 \\
203\end{array}$ & $\begin{array}{l}46.60 \\
2.00 \mathrm{~F}-09\end{array}$ & D & $\begin{array}{l}\text { THR ESH } \\
.10900\end{array}$ & AERE $\dot{E}^{23765}$ & 82064 \\
\hline P B & 206 & $(N, T)$ & $\begin{array}{l}\text { TL } \\
\text { TL }\end{array}$ & $\begin{array}{l}204 \\
204\end{array}$ & $\begin{array}{l}3.77 \\
9.00 E-10\end{array}$ & $\mathbf{Y}$ & $\begin{array}{l}\text { THRESH } \\
.30000\end{array}$ & TISO7 & 82067 \\
\hline PB & 207 & $(N, P)$ & $\begin{array}{l}\mathrm{TL} \\
\mathrm{TL}\end{array}$ & $\begin{array}{l}207 \\
207\end{array}$ & $3.00 E-06$ & & THRE SH & & 82073 \\
\hline P B & 207 & $(N, N P+D)$ & $\begin{array}{l}T L \\
T L\end{array}$ & $\begin{array}{l}206 \\
206\end{array}$ & $\begin{array}{l}4.20 \\
3.00 E=06\end{array}$ & $M$ & $\begin{array}{r}\text { THR ESH } \\
.54400\end{array}$ & AERE & 82075 \\
\hline
\end{tabular}




\begin{tabular}{|c|c|c|c|c|c|c|c|c|c|}
\hline P B & 207 & $(N, N A)$ & $\begin{array}{l}H G \\
H G\end{array}$ & $\begin{array}{l}203 \\
203\end{array}$ & $\begin{array}{l}46.60 \\
2.00 E-09\end{array}$ & D & $\begin{array}{l}\text { THRESH } \\
.10900\end{array}$ & $\triangle A E R E$ & 32076 \\
\hline$P B$ & 208 & $(N, 2 N)$ & $\begin{array}{l}P B \\
P B\end{array}$ & $\begin{array}{l}207 \\
207\end{array}$ & $3.00 E-0 t$ & & THRE SH & & 82031 \\
\hline PB & 203 & $(N, P)$ & $\begin{array}{l}\text { TL } \\
\text { TL }\end{array}$ & $\begin{array}{l}208 \\
208\end{array}$ & $\begin{array}{l}3.053 \\
3.00 E-06\end{array}$ & $M$ & $\begin{array}{l}\text { THRESH } \\
.59400\end{array}$ & $T_{M 102}^{3.38539}$ & 8208.3 \\
\hline$P B$ & 208 & $(N, A)$ & $\begin{array}{l}H G \\
H G\end{array}$ & $\begin{array}{l}205 \\
205\end{array}$ & $\begin{array}{l}5.2 \\
3.00 E-06\end{array}$ & M & $\begin{array}{l}\text { THRESH } \\
.64000\end{array}$ & TISO7 & 82084 \\
\hline$P B$ & 208 & $(N, N P+D)$ & $\begin{array}{l}T L \\
T L\end{array}$ & $\begin{array}{l}207 \\
207\end{array}$ & 3. $00 F-0 t$ & & THR F SH & & 32085 \\
\hline P B & 208 & $(N, T)$ & $\begin{array}{l}T L \\
T L\end{array}$ & $\begin{array}{l}20 t \\
206\end{array}$ & * $3.00 E-05$ & & THRESH & & 82087 \\
\hline
\end{tabular}


table displays the two title cards that precede the neutron and gamma-ray data, where appropriate, for each reaction. Definitions and format specifications for the items contained in the title cards are given in the Appendix. The thermal cross section (group 100) was computed in the following way. For those reactions for which $2200 \mathrm{~m} / \mathrm{s}$ cross sections were available, either from the ENDF/B-IV files or the Chart of the Nuclides (Twelfth Edition, 1977 Revision), the thermal cross sections was taken as $(\sqrt{ } / 2) \times \sigma_{2200}$, the average value for a $1 / v$ absorber at $293 \mathrm{~K}$. In cases where the $2200 \mathrm{~m} / \mathrm{s}$ data were not easily avallable, the group 99 cross section was assigned an energy of $0.469 \mathrm{eV}$, corresponding to the lethargy midpoint (16.875) of the group. Assuming a $1 / v$ behavior, the average cross section at $293 \mathrm{~K}$ is then equal to $\sigma_{99} \times 3.84$.

\section{RADIOACTIVE DECAY DATA}

The half-1ives assigned to the radioactive products were those shown in the Twelfth Edition of the Chart of the Nuclides (1977 Revision). In cases where a reaction led to isomeric states, and the branching ratio was not known, no halflife value was given, and the reaction was flagged with an asterisk (see Appendix). The latter situation was true for essentially all of the reactions calculated with the THRESH code. Hence, a large number of reactions have data only for neutron cross sections and lower limits for the MPC.

The gamma-ray intensitities were derived, except for ${ }^{50} \mathrm{~V}$, from the Jülich Jibrary of gamma-ray and X-ray lines for 1313 radionuclides. 5,6 Although not used by us, the Jülich file also gives data on half-1ives, parent and daughter nuclides, generating reactions, and escape lines associated with pair production processes. A code was written to bin the discrete intensities (photons/100 dis) into the 25-group structure shown in Table III. Where the file contained relative intensity data, we disregarded the entry. 'It is of interest to note that a much expanded and revised gamma-ray data file for 2055 radionuclides, GAMDAT-78, 7 has just been made available by the Jülich group. Our experience with the Jülich data has been very satisfactory, based on checks we have made for several radioisotopes. We recommend that the GAMDAT-78 compilation be used for future work until such time as the ENDF/B gamma-ray data is much more complete, and retrieval codes have been written to translate the information into a readily usable form. The GAMDAT-78 file is quite comprehensive -- approximately 48000 lines for 2055 radiolsotopes. 
TABLE III

DECAY ENERGY GROUP STRUCTURE

GR OUP

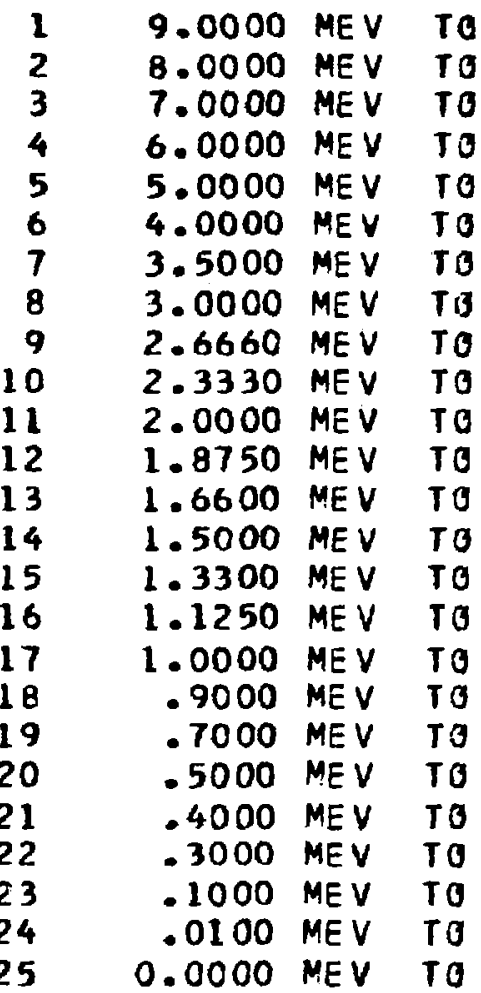

ENERGY RANGE

$$
\begin{aligned}
& 10.0000 \mathrm{MEV} \\
& 9.0000 \mathrm{MEV} \\
& 8.0000 \mathrm{MEV} \\
& 7.0000 \mathrm{MEV} \\
& 6.0000 \mathrm{MEV} \\
& 5.0000 \mathrm{MEV} \\
& 4.0000 \mathrm{MEV} \\
& 3.500 \mathrm{MEV} \\
& 3.0000 \mathrm{MEV} \\
& 2.6660 \mathrm{MEV} \\
& 2.3330 \mathrm{MEV} \\
& 2.0000 \mathrm{MEV} \\
& 1.8750 \mathrm{MEV} \\
& 1.6600 \mathrm{MEV} \\
& 1.5000 \mathrm{MEV} \\
& 1.3300 \mathrm{MEV} \\
& 1.1250 \mathrm{MEV} \\
& 1.0000 \mathrm{MEV} \\
& .9000 \mathrm{MEV} \\
& .7000 \mathrm{MEV} \\
& .5000 \mathrm{MEV} \\
& .4000 \mathrm{MEV} \\
& .3000 \mathrm{MEV} \\
& .1000 \mathrm{MEV} \\
& .0100 \mathrm{MEV}
\end{aligned}
$$

ENERGY WIDTH

$$
\begin{aligned}
& 1.0000 \mathrm{MEV} \\
& 1.0000 \mathrm{MEV} \\
& 1.0000 \mathrm{MEV} \\
& 1.0000 \mathrm{MEV} \\
& 1.0000 \mathrm{MEV} \\
& 1.0000 \mathrm{MEV} \\
& .5000 \mathrm{MEV} \\
& .5000 \mathrm{MEV} \\
& .3340 \mathrm{MEV} \\
& .3330 \mathrm{MEV} \\
& .3330 \mathrm{MEV} \\
& .1250 \mathrm{MEV} \\
& .2150 \mathrm{MEV} \\
& .1600 \mathrm{MEV} \\
& .1700 \mathrm{MEV} \\
& .2050 \mathrm{MEV} \\
& .1250 \mathrm{MEV} \\
& .1000 \mathrm{MEV} \\
& .2000 \mathrm{MEV} \\
& .2000 \mathrm{MEV} \\
& .1000 \mathrm{MEV} \\
& .1000 \mathrm{MEV} \\
& .2000 \mathrm{MEV} \\
& .0900 \mathrm{MEV} \\
& .0100 \mathrm{MEV}
\end{aligned}
$$


The gamma energy ( $\mathrm{MeV}$ ) per disintegration was computed from the data in the Juilich library. This was done by summing over the products of gamma-ray energy and intensity prior to binning into the 25-group structure. For the non-gamma energy entries, we drew heavily on the published 1iterature, especially those reported by Nichols ${ }^{8}$ and Kocher. ${ }^{9}$ Because the Jülich library accounts for both gamma rays (including annihilation radiation) and $x$ rays, we needed only the energies of the Auger and conversion electrons and alphas. For the 50 reactions for which non-gamma energies were not found in the literature, we used the Table of Isotopes. ${ }^{10}$ In view of the limited time avallable, we assumed the mean beta energy to be 0.4 times the endpoint energy and ignored the contribution from Auger and conversion electrons. The neglect of the latter contribution is wot serious since this component is usually very small compared to the total (gamma + non-gamma) energy release.

\section{MAXIMUM PERMISSIBLE CONCENTRATIONS}

The Code of Federal Regulations ${ }^{11}$ specifies limits, in air and water, for radioactivity in effluents to unrestricted areas. We used the latter values for air; where MPCs for soluble and Insoluble forms were given, the lower value was chosen. For isotopes not assigned spectflc MPC values, the code recommends numbers whose magnitudes depend on whether (1) the half-1ife is less than 2 hours, (2) the half-life is greater than 2 hours, and (3) the decay is by alpha emission or spontaneous fission. In those cases where the reaction leads to metastable states and the branching ratio is unknown, the lower figure for the MPC was used.

\section{APPENDIX}

\section{GAMMON FORMAT SPECIFICATIONS}

\section{Title Card 1}

Target symbol

\section{$\underline{\text { Column }} \underline{\text { Format }}$ Content}
Reaction
Target symbol
4-6
A1
A3
$9-16$

\author{
Element - H, PU, etc. \\ Blank \\ Mass - 1, 239, etc. \\ $(N, G),(N, 2 N),(N, N P+D)$, etc.
}

$1-2$ 


\begin{tabular}{|c|c|c|c|}
\hline Product symbol & $\begin{array}{l}19-20 \\
21 \\
22-24 \\
25\end{array}$ & $\begin{array}{l}\text { A2 } \\
\text { A1 } \\
\text { A3 } \\
\text { A1 }\end{array}$ & $\begin{array}{l}\text { Element } \\
\text { Blank } \\
\text { Mass } \\
\text { M for isomeric state }\end{array}$ \\
\hline Half-1Ife & $27-36$ & $F$ or $E 10.3$ & $\begin{array}{l}\text { Numerical value. An asterisk In } \\
\text { column } 28 \text { indicates that the half- } \\
\text { life of the reaction product can- } \\
\text { not be unfquely specified because } \\
\text { of the existence of important } \\
\text { Isomeric states. } \\
\text { Half-1ife units: } \mathrm{S} \text { - second } \\
\text { M - minute } \\
\text { H - hour } \\
\text { D - cay } \\
\text { Y - year }\end{array}$ \\
\hline Data source & $43-53$ & $A 6, A 5$ & ENDF/B-IV, THRESH, etc. \\
\hline Gamma MeV/dis & $54-61$ & F8.5 & $\begin{array}{l}\text { MeV of ganma radiation per disin- } \\
\text { tegration. Blank if * in column } \\
28 \text {. }\end{array}$ \\
\hline $\begin{array}{l}\text { Target identifier and } \\
\text { reaction type }\end{array}$ & $\begin{array}{l}73-74 \\
75-76 \\
77\end{array}$ & $\begin{array}{l}\text { I2 } \\
\text { I2 } \\
\text { I1 }\end{array}$ & 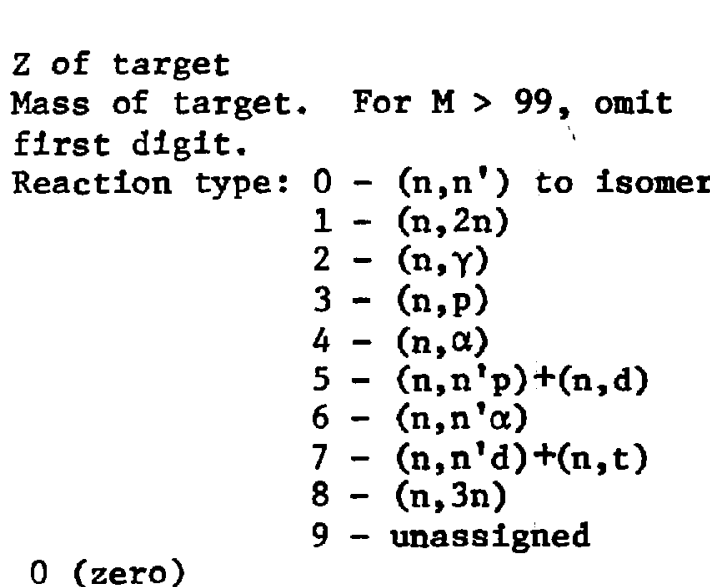 \\
\hline
\end{tabular}

Title Card 2

Product symbol

19-25 A7

MPC

27-35 E9.2

See Title Card 1.

MPC reference

$37-38$

A2

Maximum permissible concentration in units of $\mu \mathrm{Cl} / \mathrm{ml}$.

Unless otherwise noted, values are from Ref. 11. The entry DK denotes data are from Ref. 13.

Non-gamma MeV/dis

$43-50$

F8.5

MeV per disintegration, excluding photons. Blank if * in column 28 of Title Card 1. 


$$
\begin{aligned}
& \text { AERE - Ref. } 8 \\
& \text { TM102 - Ref. } 9 \\
& \text { TIS07 - Ref. } 10 \\
& \text { MEDLI - Ref. } 12 \\
& \text { DK - Ref. } 13 \\
& \text { EN223 - Ref. } 14
\end{aligned}
$$

Cards 3-19. 100-Group Neutron Cross Sections

$\begin{array}{lll}\text { Cross sections } & 1-72 & 6 \mathrm{E} 12.5\end{array}$

Target identifier and reaction type

73-77

I5

78

$79-80$
Blank

I2
See Title Card 1

1 to 17

Cards 20-23. 25-Group Gamma Intensities

These cards not present if gamma MeV/dis 0.0 or there is an * In column 28 of Title Card 1.

Intensities

$1-70$

7F10. 5

Photons per 100 disintegrations

Product symbol

$73-79$

A7

See Title Card 1

\section{REFERENCES}

1. R. W. Roussin, D. W. Muir, and M. E. Battat, "MONTAGE-400: 100 Group Neutron Activation Cross-Section Data for Fusion Reactor Structure and Coolant Materials," Radiation Shielding Information Center, Oak Ridge National Laboratory, Library Collection DLC-33/MONTAGE-400 (Oct. 1978).

2. H. J. Willenberg and W. E. Bickford, "Safety Aspects of Activation Products in a Compact Tokamak Fusion Power Plant," Pacific Northwest Laboratory report PNL-2823 (UC-2OE) (Oct. 1978).

3. S. Pearlstein, "Neutron-Induced Reactions in Medium Mass Nuclei," J. Nuc1. Energy 27, 81 (1973).

4. P. G. Young and E. D. Arthur, "GNASH: A Preequilibrium Statistical NuclearModel Code for Calculation of Cross Sections and Emission Spectra," Los Alamos Scientific Laboratory report LA-6947 (Nov. 1977).

5. G. Erdtmann and W. Soyka, "The Gamma-Ray Lines of Radionuclides, Ordered by Atomic and Mass Number: Part I. $\mathrm{Z}=2-57$ (Helium-Lanthanum)," J, of Radioanalytical Chem. 26, 375 (1975).

6. G. Erdtmann and W. Soyka, "The Gamma-Ray Lines of Radionuclides, Ordered by Atomic and Mass Number:Part II. $\mathrm{Z}=$ 58-100 (Cerium-Fermium)," J. of Radioanalytical Chem. 27, 137 (1975). 
7. G. Erdtmann and W. Soyka, "The Radionuclide Gamma-Ray Data File 'GAMAT-78'," Kernforschungsanlage Julich GmbH special publication JUL-Spez-34 (to be published in 1979).

8. A. L. Nichols, "Radioactive-Nuclide Decay Data for Reactor Calculations: Activation Products and Related Isotopes," Atomlc Energy Research Estab1ishment, Harwell, report AWRE-R8903 (1978).

9. D. C. Kocher, "Nuclear Decay Data for Radionuclides Occurring in Routine Releases from Nuclear Fuel Cycle Facilities," Oak Ridge National Laboratory report ORNL/NUREG/TM-102 (Aug. 1977).

10. "Table of Isotopes - Seventh Edition," by staff members of the Nuclear Science Diviston, Lawrence Berkeley Laboratory, Berkeley (Wiley Intersclence, New York, 1978).

11. Code of Federa1 Regulations, 10CFR20 - Appendix B (U. S. Government Printing Office, Washington, D.C., 1979).

12. "MEDLIST: RADIONUCLIDE Radiation Data from the Evaluated Nuclear Structure Data File (ENSDF) for interest in Medical, Health Physics, Nuclear Power, Environmental Impact, and Industrial App1ications," contributed by Nuclear Data Project, Oak Ridge National Laboratory, Radiation Shielding Information Center Library Collection DLC-46/MEDLIST (March 1977).

13. Donald J. Dudziak and R. A. Krakowski, "Radioactivity Induced in a ThetaPinch Fusion Reactor," Nuc1. Tech. 25, 32 (1975).

14. T. R. England and R. E. Schenter, "ENDF/B-IV Fission-Product Files: Summary of Major Nuclide Data," Los Alamos Scientific Laboratory report LA-6116-MS (ENDF-223) (Oct. 1975). 